\title{
On the origin of nitrogen
}

\author{
L. S. Pilyugin ${ }^{1}$, T. X. Thuan ${ }^{2}$, and J. M. Vílchez ${ }^{3}$
}

\author{
1 Main Astronomical Observatory of National Academy of Sciences of Ukraine, 27 Zabolotnogo str., 03680 Kiev, Ukraine \\ 2 Astronomy Department, University of Virginia, Charlottesville, VA 22903, USA \\ 3 Instituto de Astrofísica de Andalucía, Apdo, 3004, 18080 Granada, Spain
}

Received 30 May 2002 / Accepted 30 September 2002

\begin{abstract}
The problem of the origin of nitrogen is considered within the framework of an empirical approach. The oxygen abundances and nitrogen to oxygen abundances ratios are derived in $\mathrm{H}$ II regions of a number of spiral galaxies through the recently suggested $P$-method using more than six hundred published spectra. The $\mathrm{N} / \mathrm{O}-\mathrm{O} / \mathrm{H}$ diagram for $\mathrm{H}$ II regions in irregular and spiral galaxies is constructed. It is found that the N/O values in $\mathrm{H}$ II regions of spiral galaxies of early morphological types are higher than those in $\mathrm{H}_{\text {II }}$ regions with the same metallicity in spiral galaxies of late morphological types. This suggests a long-time-delayed contribution to the nitrogen production. The N/O ratio of a galaxy can then be used as an indicator of the time that has elapsed since the bulk of star formation occurred, or in other words of the nominal "age" of the galaxy as suggested by Edmunds \& Pagel more than twenty years ago. The scatter in N/O values at a given $\mathrm{O} / \mathrm{H}$ can be naturally explained by differences in star formation histories in galaxies. While low-metallicity dwarf galaxies with low N/O do not contain an appreciable amount of old stars, low-metallicity dwarf galaxies with an appreciable fraction of old stars have high N/O. Consideration of planetary nebulae in the Small Magellanic Cloud and in the Milky Way Galaxy suggests that the contribution of low-mass stars to the nitrogen production is significant, confirming the conclusion that there is a long-time-delayed contribution to the nitrogen production.
\end{abstract}

Key words. galaxies: abundances - galaxies: ISM - galaxies: spiral - galaxies: irregular

\section{Introduction}

The origin of nitrogen has always been one of the central problems of the theory of chemical evolution of galaxies. The theoretical stellar yields of nitrogen have been computed by many investigators (Renzini \& Voli 1981; Maeder 1992; Marigo et al. 1996, 1998, 2001; van den Hoek \& Groenewegen 1997; Boothroyd \& Sackmann 1999; Rauscher et al. 2002; Meynet \& Maeder 2002a,b; among others). However, the results are not undisputed. Therefore, the "empirical" approach to study the origin of nitrogen, i.e. the comparison of the predictions of models of chemical evolution of galaxies computed with different assumptions for nitrogen yields with observed $\mathrm{N}$ abundances, has been widely used (Edmunds \& Pagel 1978; Lequeux et al. 1979; Serrano \& Peimbert 1983; Matteucci \& Tosi 1985; Matteucci 1986; Garnett 1990; Pilyugin 1992, 1993, 1999; Vila-Costas \& Edmunds 1993; Marconi et al. 1994; Thuan et al. 1995; Thurston et al. 1996; Kobulnicky \& Skillman 1996, 1998; van Zee et al. 1998a; Izotov \& Thuan 1999; Coziol et al. 1999; Henry et al. 2000; Contini et al. 2002; among others). The interpretation of the observed N/O vs. O/H diagram is at the base of the empirical approach.

Send offprint requests to: L. S. Pilyugin, e-mail: pilyugin@mao.kiev.ua
Thanks to the work of Thuan et al. (1995), Kobulnicky \& Skillman (1996), van Zee et al. (1997), Izotov \& Thuan (1999), and others, the N/O vs. O/H diagram appears to be well established in the low-metallicity range. In the low-metallicity range, it has significantly changed during the last decade. There is no commonly accepted interpretation of the present-day N/O vs. O/H diagram. Izotov \& Thuan (1999) have concluded that massive stars are responsible for the nitrogen abundances observed in low-metallicity blue compact galaxies, while Henry et al. (2000) have found that intermediate-mass stars make a dominant contribution to the nitrogen production. As noted by Henry et al. (2000), a major problem with N/O ratios has been to try to explain the spread in $\mathrm{N} / \mathrm{O}$ at a given $\mathrm{O} / \mathrm{H}$. Close examination of the suggested explanations of the origin of the scatter in the present-day $\mathrm{N} / \mathrm{O}-\mathrm{O} / \mathrm{H}$ diagram shows that existing explanations are not satisfactory (see below). This provides the motivation for this study.

We use also here an empirical approach to study the origin of nitrogen. Why should we hope that this attempt would be more successful than previous ones based on the empirical approach in establishing the origin of nitrogen? Most studies on the origin of nitrogen have concentrated on low-luminosity dwarf galaxies. Van Zee et al. (1998a) have shown that the use of low-metallicity $\mathrm{H}$ II regions at the periphery of spiral galaxies for investigation of the origin of nitrogen provides 
some advantages in comparison to $\mathrm{H}$ II regions in dwarf irregular galaxies. In previous studies, the oxygen abundances in high-metallicity $\mathrm{H}$ II regions, where [OIII] $\lambda 4363$ line is usually not available, were derived using an empirical method (usually the $R_{23}-$ method suggested by Pagel et al. 1979). Recently it has been shown (Pilyugin 2000, 2001a,b) that the oxygen abundance derived with the $R_{23}$-method produces a systematic error depending on the excitation parameter $P$ : the $R_{23}-$ method provides more or less realistic oxygen abundances in high-excitation $\mathrm{H}_{\mathrm{II}}$ regions, but yields overestimated oxygen abundances in low-excitation $\mathrm{H}$ II regions. This is in agreement with the result of Kinkel \& Rosa (1994), who showed the need of lowering all $\mathrm{H}$ II region abundances obtained with the $R_{23}$ calibration of Edmunds \& Pagel (1984) for intrinsic solar-like $\mathrm{O} / \mathrm{H}$ values and above. Castellanos et al. (2002) also found that the $R_{23}-$ method yields an overestimated oxygen abundances in low-excitation $\mathrm{H}_{\text {II }}$ regions. Thus, the high-metallicity part of the $\mathrm{N} / \mathrm{O}$ vs. $\mathrm{O} / \mathrm{H}$ diagram in previous studies can be highly uncertain. A new way of oxygen abundance determination in H II regions (called the $P$-method) was suggested by Pilyugin (2001a). It was demonstrated that the oxygen abundances derived with the $P$-method are as reliable as the ones derived with the classic $T_{\mathrm{e}}-$ method (Pilyugin 2001a,b). Thus, we will be able to construct a more realistic high-metallicity part of the $\mathrm{N} / \mathrm{O}$ vs. $\mathrm{O} / \mathrm{H}$ diagram in the present study.

Traditionally, the problem of the nitrogen production is discussed in the framework of primary versus secondary processes. Since an empirical approach is adopted in the present study, the problem of the nitrogen production will be discussed in the framework of metallicity-independent versus metallicitydependent yields. The metallicity dependence of the yield of element $x$ does not necessary imply that element $x$ is a secondary one. Instead it can be result of stellar mass loss which can affect both stellar evolution and the production of element $x$. The dependence of stellar mass loss on metallicity leads to a metallicity dependence of the yield of element $x$ even if that element is primary. Indeed, Maeder (1992) predicts a metallicity dependence of the yield of the primary element oxygen as the result of a metallicity-dependent mass loss process.

The determination of oxygen abundances and nitrogen to oxygen abundance ratios in high-metallicity $\mathrm{H}$ II regions of galaxies and the construction of an accurate $\mathrm{N} / \mathrm{O}-\mathrm{O} / \mathrm{H}$ diagram for $\mathrm{H}_{\mathrm{II}}$ regions in irregular and spiral galaxies is described in Sect. 2 . The nitrogen production sites derived from the analysis of the $\mathrm{N} / \mathrm{O}-\mathrm{O} / \mathrm{H}$ diagram and observational data of other kinds are reported in Sect. 3. A discussion is presented in Sect. 4. Section 5 is a brief summary.

\section{The $\mathrm{N} / \mathrm{O}-\mathrm{O} / \mathrm{H}$ diagram}

\subsection{Low-metallicity $\mathrm{H}$ II regions in irregular galaxies}

Generally, the precision of the oxygen abundance determination in oxygen-poor $\mathrm{H}_{\mathrm{II}}$ regions with bright emission lines in irregulars is higher than in oxygen-rich $\mathrm{H}_{\text {II }}$ regions in spirals. However, many irregular galaxies have no bright $\mathrm{H}$ II regions with readily measured emission lines. As was noted by Hidalgo-Gámez \& Olofsson (1998) the uncertainties in the

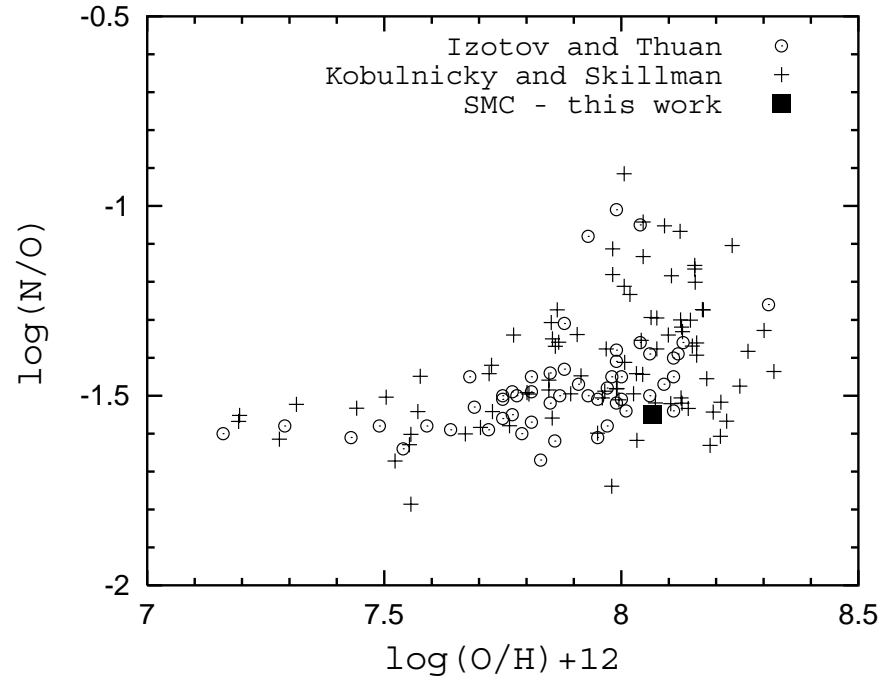

Fig. 1. The $\mathrm{N} / \mathrm{O}$ versus $\mathrm{O} / \mathrm{H}$ diagram for $\mathrm{H}$ II regions in irregular galaxies. The open circles are data from Izotov \& Thuan (1999), the pluses are data from Kobulnicky \& Skillman (1996). The filled square denotes the Small Magellanic Cloud (this work).

intensity of the [OIII] $\lambda 4363$ line in spectra of $\mathrm{H}$ II regions in dwarf irregular galaxies reported in the literature fluctuate between $11 \%$ and $120 \%$. Then the precision of the oxygen abundance determination in oxygen-poor irregulars without $\mathrm{H}$ II regions with bright emission lines is rather low (Pilyugin 2001c). Therefore we did not collect all the available data but instead have considered only two data sets.

Kobulnicky \& Skillman (1996) have selected from the literature a large sample (72 measurements in 60 galaxies) of spectroscopic measurements in metal-poor galaxies $(12+$ $\log (\mathrm{O} / \mathrm{H})<\sim 8.4)$ and have recomputed the $\mathrm{O}$ and N/O abundances in a self-consistent manner. Their data are shown in Fig. 1 with pluses. Izotov \& Thuan (1999) have presented O and $\mathrm{N}$ abundances derived from high-quality spectroscopic observations of 54 supergiant $\mathrm{H}$ II regions in 50 low-metallicity blue compact galaxies with oxygen abundances $12+\log (\mathrm{O} / \mathrm{H})$ between 7.1 and 8.3. These data are presented in Fig. 1 with open circles.

The observed N/O-O/H diagram in the low-metallicity range has appreciably changed over the last decade. The early N/O-O/H diagram (see Fig. 7 in Garnett 1990, Fig. 1 in Pilyugin 1992) consisted of a cloud of points with a more or less constant upper envelope of $\mathrm{N} / \mathrm{O}$, and with the spread in $\mathrm{N} / \mathrm{O}$ at a given $\mathrm{O} / \mathrm{H}$ sligtly decreasing with increasing $\mathrm{O} / \mathrm{H}$. In contrast, the scatter in $\mathrm{N} / \mathrm{O}$ at a given $\mathrm{O} / \mathrm{H}$ in the present $\mathrm{N} / \mathrm{O}$ $\mathrm{O} / \mathrm{H}$ diagram is extremelly small at low $\mathrm{O}$ abundances. The scatter becomes large only when the metallicity exceeds a certain value $(12+\log (\mathrm{O} / \mathrm{H})$ around 7.6), and up to $\mathrm{O}$ abundances around $12+\log (\mathrm{O} / \mathrm{H})=8.3$ the lower envelope of $\mathrm{N} / \mathrm{O}$ is equal to the N/O value at low $\mathrm{O}$ abundances, Fig. 1, (see also Fig. 2 in Thuan et al. 1995, Fig. 15 in Kobulnicky \& Skillman 1996, Fig. 2 in Izotov \& Thuan 1999). 


\subsection{HII regions in the Small Magellanic Cloud}

The Small Magellanic Cloud is a well observed dwarf irregular galaxy. Examination of this galaxy can clarify the chemical enrichment history in irregulars. The $\mathrm{H}_{\mathrm{II}}$ regions in the Small Magellanic Cloud were observed by a number of investigators. Their oxygen and nitrogen abundances were recomputed here in the same way with the classic $T_{\mathrm{e}}$-method, using published spectra. The electron temperature within the [OIII] zone is derived from equation (Pagel et al. 1992)

$$
\begin{aligned}
t_{3}=1.432 /[ & \log R-0.85+0.03 \log t_{3} \\
+ & \left.\log \left(1+0.0433 x t_{3}^{0.06}\right)\right],
\end{aligned}
$$

where $R=I_{[\mathrm{OIII}] \lambda 4959+\lambda 5007} / I_{[\mathrm{OIII}] \lambda 4363}$. Using the obtained value of $t_{3}$, the oxygen and nitrogen abundances were derived from the following expressions (Pagel et al. 1992)

$\frac{\mathrm{N}}{\mathrm{O}}=\frac{\mathrm{N}^{+}}{\mathrm{O}^{+}}$,

$\log \left(\mathrm{N}^{+} / \mathrm{O}^{+}\right)=\log \frac{I_{[\mathrm{NII}] \lambda 6548+\lambda 6584}}{I_{[\mathrm{OII}] \lambda 3726+\lambda 3729}}+0.307-\frac{0.726}{t_{2}}$

$\frac{\mathrm{O}}{\mathrm{H}}=\frac{\mathrm{O}^{+}}{\mathrm{H}^{+}}+\frac{\mathrm{O}^{++}}{\mathrm{H}^{+}}$

$$
\begin{array}{r}
12+\log \left(\mathrm{O}^{++} / \mathrm{H}^{+}\right)=\log \frac{I_{[\mathrm{OIII}] \lambda 4959+\lambda 5007}}{I_{\mathrm{H}_{\beta}}}+6.174 \\
+\frac{1.251}{t_{3}}-0.55 \log t_{3},
\end{array}
$$

$$
\begin{aligned}
& 12+\log \left(\mathrm{O}^{+} / \mathrm{H}^{+}\right)=\log \frac{I_{[\mathrm{OII}] \lambda 3726+\lambda 3729}}{I_{\mathrm{H}_{\beta}}}+5.890 \\
& +\frac{1.676}{t_{2}}-0.40 \log t_{2}+\log (1+1.35 x), \\
& x=10^{-4} n_{\mathrm{e}} t_{2}^{-1 / 2},
\end{aligned}
$$

where $n_{\mathrm{e}}$ is the electron density in $\mathrm{cm}^{-3}, t_{2}=t_{[\mathrm{NII}]}$ is the electron temperature in units of $10^{4} \mathrm{~K}$. We adopt $t_{2}=t_{[\mathrm{NII}]}=t_{[\mathrm{OII}]}$. The value of $n_{\mathrm{e}}$ is adopted to be equal to $100 \mathrm{~cm}^{-3}$ for all $\mathrm{H}_{\mathrm{II}}$ regions. The $t_{2}-t_{3}$ relation was taken from Garnett (1992):

$t_{2}=0.7 t_{3}+0.3$.

The results are presented in Table 1 . The name of the $\mathrm{H}$ in region is listed in Col. 1. The oxygen abundance is reported in Col. 2. The nitrogen to oxygen abundance ratio is given in Col. 3 . The reference is listed in Col. 4. Examination of Table 1 shows that the oxygen and nitrogen abundances in the interstellar medium of the Small Magellanic Cloud are well defined; oxygen and nitrogen abundances derived with spectroscopic data from different studies are in good agreement. We note that the nitrogen to oxygen ratio in the $\mathrm{H}$ II regions of the Small Magellanic Cloud is very close to that in the $\mathrm{H}$ in regions of the most oxygen-poor blue compact galaxies, Fig. 1.
Table 1. The oxygen and nitrogen abundances in $\mathrm{H}_{\text {II }}$ regions of the Small Magellanic Cloud recomputed here with the classic $T_{\mathrm{e}}-$ method using published spectra. The name of the $\mathrm{H}_{\text {II }}$ region is listed in Col. 1. The oxygen abundance is reported in Col. 2 . The nitrogen to oxygen abundance ratio is given in Col. 3. The reference is listed in Col. 4.

\begin{tabular}{llll}
\hline \hline & & & \\
H II region & $12+\log (\mathrm{O} / \mathrm{H})$ & $\log (\mathrm{N} / \mathrm{O})$ & reference \\
\hline N66a & 8.081 & -1.525 & Duf75 \\
N83a & 8.122 & -1.582 & Duf75 \\
NGC 346 I & 8.034 & -1.520 & PTP76 \\
NGC 346 II & 8.068 & -1.633 & PTP76 \\
NGC 356 I & 7.980 & -1.678 & PTP76 \\
NGC 456 I & 8.087 & -1.502 & PTP76 \\
N12a & 8.156 & -1.509 & DH77 \\
N12b & 8.018 & -1.590 & DH77 \\
N22 & 8.206 & -1.665 & DH77 \\
N25 & 8.037 & -1.551 & DH77 \\
N66NW & 8.098 & -1.603 & DH77 \\
N66SE & 8.114 & -1.465 & DH77 \\
N76E & 7.986 & -1.321 & DH77 \\
N76SW & 7.902 & -1.418 & DH77 \\
N81 & 8.120 & -1.513 & DH77 \\
N83 & 8.105 & -1.597 & DH77 \\
N88 & 7.971 & -1.591 & DH77 \\
N90 & 8.094 & -1.546 & DH77 \\
N13 & 8.117 & -1.547 & Pag78 \\
NGC 346 A & 8.056 & -1.598 & PPR00 \\
NGC 346 B & 8.033 & -1.593 & PPR00 \\
MEAN & $8.066 \pm 0.068$ & $-1.550 \pm 0.080$ & \\
\hline \hline
\end{tabular}

List of references:

DH77 - Dufour \& Harlow (1977); Duf75 - Dufour (1975); Pag78 Pagel et al. (1978); PPR00 - Peimbert et al. (2000); PTP76 - Peimbert \& Torres-Peimbert (1976).

\subsection{High-metallicity $\mathrm{H}$ II regions in spiral galaxies}

The N/O vs. O/H diagram for $\mathrm{H}_{\mathrm{II}}$ regions in spiral galaxies has been constructed in a number of studies (Matteucci 1986; Vila-Costas \& Edmunds 1993; van Zee et al. 1998a; Henry et al. 2000; among others). In those studies, the oxygen abundances in high-metallicity $\mathrm{H}_{\text {II }}$ regions of spiral galaxies are derived using an empirical $R_{23}$-calibration, inducing a large uncertainty in the $\mathrm{N} / \mathrm{O}$ vs. $\mathrm{O} / \mathrm{H}$ diagram as mentioned in the Introduction. Here we attempt to construct a more precise $\mathrm{N} / \mathrm{O}$ vs. $\mathrm{O} / \mathrm{H}$ diagram for $\mathrm{H}$ II regions in spiral galaxies. The oxygen abundances and N/O abundance ratios in high-metallicity $\mathrm{H}$ II regions, where $\lambda 4363$ is not available, are determined with the following algorithm. The oxygen abundance is determined from the expression (Pilyugin 2001a)

$$
12+\log (\mathrm{O} / \mathrm{H})_{P}=\frac{R_{23}+54.2+59.45 P+7.31 P^{2}}{6.07+6.71 P+0.371 P^{2}+0.243 R_{23}} .
$$

The following notations have been adopted here: $R_{2}=$ $I_{[\mathrm{OII}] \lambda 3727+\lambda 3729} / I_{\mathrm{H} \beta}, R_{3}=I_{[\mathrm{OIII}] \lambda 4959+\lambda 5007} / I_{\mathrm{H} \beta}, R_{23}=R_{2}+R_{3}$, $P=R_{3} / R_{23}$.

Using the value of $\mathrm{O} / \mathrm{H}$ derived from Eq. (9) and measured line intensities, Eqs. (4)-(8) can be solved for $t_{2}$. Then the N/O abundance ratio is derived from the Eqs. (2)-(3). 
Alternatively, the value of $t_{2}$ can be also found from the following expression for $t_{P}=t_{3}$ (Pilyugin 2001a)

$t_{P}=\frac{R_{23}+3.09+7.05 P+2.87 P^{2}}{9.90+11.86 P+7.05 P^{2}-0.583 R_{23}}$

and Eq. (8). The value of $t_{2}$ determined from Eqs. (8) and (10) is in good agreement with the value of $t_{2}$ derived from Eqs. (4)(9) for the majority of the $\mathrm{H}$ II regions considered. However, for $\mathrm{H}$ II regions in which most of the oxygen is in the $\mathrm{O}^{+}$stage, the two values of $t_{2}$ are not in agreement. Equations (4)-(9) give more realistic values of $t_{2}$ for these $\mathrm{H}$ II regions.

The comparison between the $(\mathrm{N} / \mathrm{O})_{T_{\mathrm{e}}}-(\mathrm{O} / \mathrm{H})_{T_{\mathrm{e}}}$ and $(\mathrm{N} / \mathrm{O})_{P^{-}}$ $(\mathrm{O} / \mathrm{H})_{P}$ diagrams for $\mathrm{H}$ II regions of the well - observed spiral galaxy M101 allows to test the reliability of the $(\mathrm{N} / \mathrm{O})_{P}$ values. The spectra of the HII regions in M101 with measured temperature-sensitive line ratios are given in a number of publications (Garnett \& Kennicutt 1994; Garnett et al. 1999; Kinkel \& Rosa 1994; McCall et al. 1985; Rayo et al. 1982; Shields \& Searle 1978; Smith 1975; Torres-Peimbert et al. 1989; van Zee et al. 1998b). The $(\mathrm{N} / \mathrm{O})_{T_{\mathrm{e}}}-(\mathrm{O} / \mathrm{H})_{T_{\mathrm{e}}}$ diagram for $\mathrm{H}$ II regions of M101 with measured temperaturesensitive line ratios is presented in Fig. 2 by open circles. Two or more independent observations of the same $\mathrm{H}$ II region are connected by a solid line. The $(\mathrm{N} / \mathrm{O})_{P}-(\mathrm{O} / \mathrm{H})_{P}$ diagram for the $\mathrm{H}$ II regions of M101 is shown in Fig. 2 by the pluses. The intensities of $I_{[\mathrm{OII}] \lambda 3727+\lambda 3729} / I_{\mathrm{H} \beta}, I_{[\mathrm{OIII}] \lambda 4959+\lambda 5007} / I_{\mathrm{H} \beta}$, and $I_{[\mathrm{NII}] \lambda 6548+\lambda 6584} / I_{\mathrm{H} \beta}$ were taken from Smith (1975), Shields \& Searle (1978), Rayo et al. (1982), McCall et al. (1985), Torres-Peimbert et al. (1989), Garnett \& Kennicutt (1994), Kinkel \& Rosa (1994), Kennicutt \& Garnett (1996), van Zee et al. (1998b), Garnett et al. (1999). Inspection of Fig. 2 shows that there is good agreement between the $(\mathrm{N} / \mathrm{O})_{T_{\mathrm{e}}}-(\mathrm{O} / \mathrm{H})_{T_{\mathrm{e}}}$ and $(\mathrm{N} / \mathrm{O})_{P}-(\mathrm{O} / \mathrm{H})_{P}$ diagrams, confirming the reliability of the $(\mathrm{N} / \mathrm{O})_{P}$ and $(\mathrm{O} / \mathrm{H})_{P}$ values.

The oxygen abundances $(\mathrm{O} / \mathrm{H})_{P}$ and nitrogen-to-oxygen abundances ratios $(\mathrm{N} / \mathrm{O})_{P}$ have been derived for $\mathrm{H}$ II regions in the following galaxies: NGC 224, NGC 253, NGC 300, NGC 598, NGC 628, NGC 753, NGC 925, NGC 1058, NGC 1232, NGC 1365, NGC 1637, NGC 2403, NGC2 805, NGC 2841, NGC 2903, NGC 2997, NGC 3031, NGC 3184, NGC 3351, NGC 4254, NGC 4258, NGC 4303, NGC 4321, NGC 4395, NGC 4501, NGC 4751, NGC 4651, NGC 4654, NGC 4689, NGC 4713, NGC 4736, NGC 5055, NGC 5194, NGC 5236, NGC 5457, NGC 6384, NGC 6946, NGC 7331, NGC 7793, and IC342. We use Eqs. (2)-(9) and more than six hundred published spectra from Alloin et al. (1981), Blair et al. (1982), Bresolin et al. (1999), Dennefeld \& Kunth (1981), Diaz et al. (1991), Dufour et al. (1980), Edmunds \& Pagel (1984), Ferguson et al. (1998), Fierro et al. (1986), Henry et al. (1992, 1994, 1996), Garnett et al. (1997), Garnett et al. (1999), Garnett \& Shields (1987), Kennicutt \& Garnett (1996), Kinkel \& Rosa (1994), Kwitter \& Aller (1981), McCall et al. (1985), Pagel et al. (1979), Rayo et al. (1982), Roy \& Walsh (1997), Searle (1971), Shields et al. (1991), Skillman et al. (1996), Smith (1975), Staufer \& Bothum (1984), Torres-Peimbert et al. (1989), van Zee et al. (1998b), Vilchez et al. (1988), and Webster \& Smith (1983). The N/O-O/H diagram for $\mathrm{H}$ II regions in spiral galaxies is shown in Fig. 3. The detailed

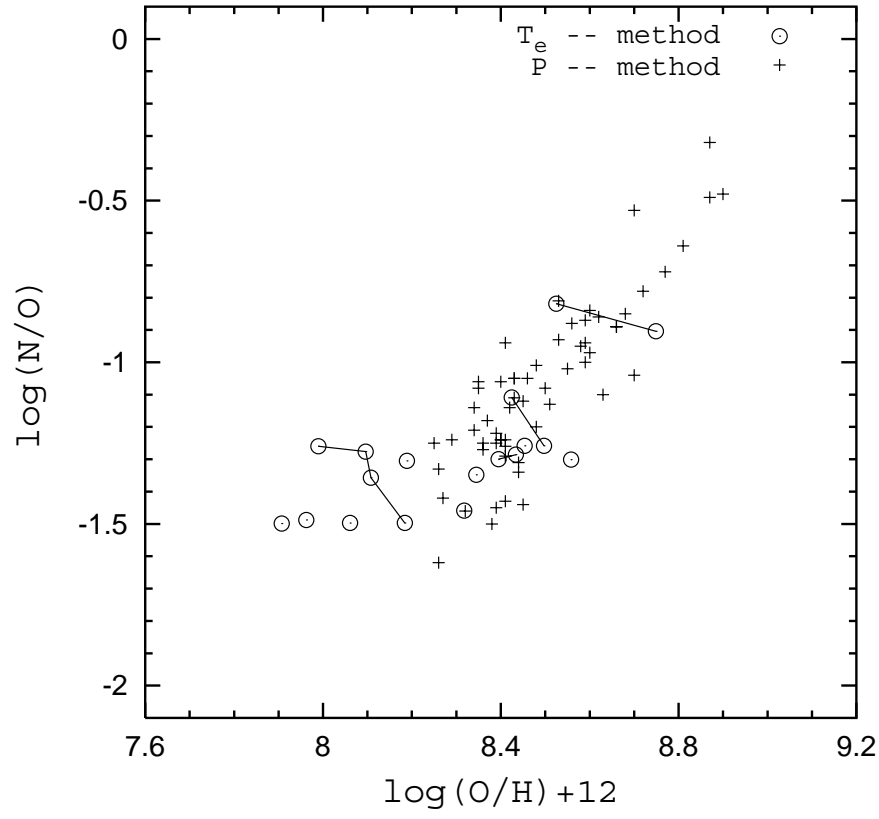

Fig. 2. The $(\mathrm{N} / \mathrm{O})_{T_{\mathrm{e}}}-(\mathrm{O} / \mathrm{H})_{T_{\mathrm{e}}}$ diagram for $\mathrm{H}_{\text {II }}$ regions of M101 with measured temperature-sensitive line ratios is shown by open circles. Two or more independent observations of the same $\mathrm{H}$ II region are connected with a solid line. The $(\mathrm{N} / \mathrm{O})_{P}-(\mathrm{O} / \mathrm{H})_{P}$ diagram for $\mathrm{H}_{\text {II }}$ regions of M101 is shown by the pluses.

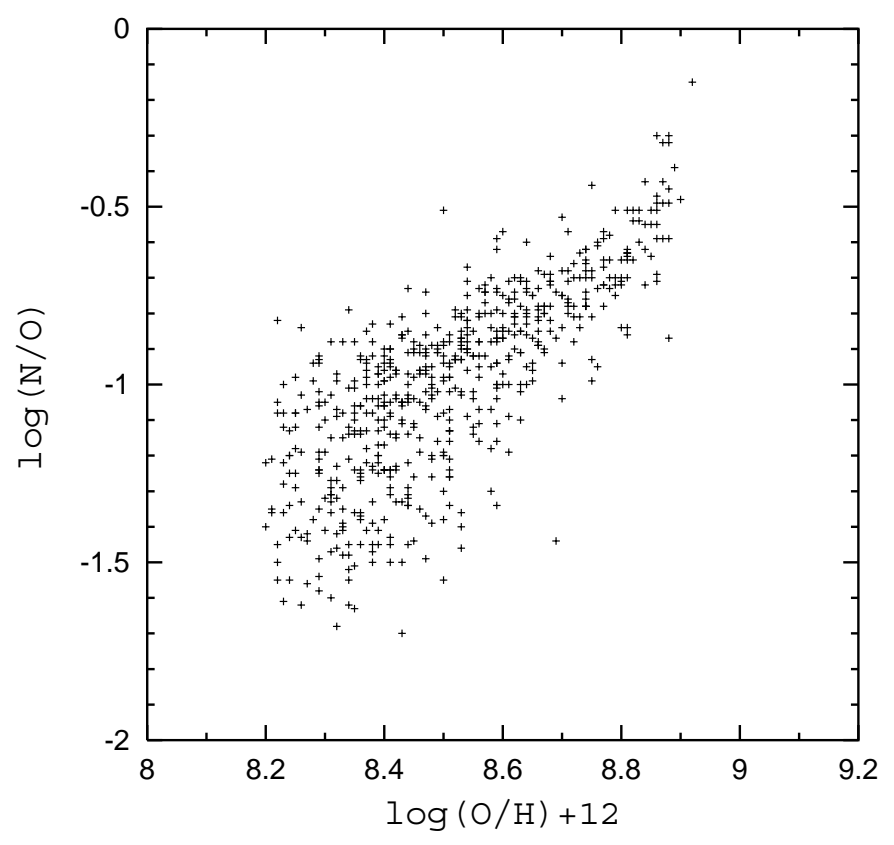

Fig. 3. The $\mathrm{N} / \mathrm{O}-\mathrm{O} / \mathrm{H}$ diagram for $\mathrm{H}$ II regions in spiral galaxies.

discussion of the radial nitrogen abundance distribution across the disks of spiral galaxies will be given elsewhere.

A prominent feature of Fig. 3 is the lack of $\mathrm{H}$ II regions with $12+\log (\mathrm{O} / \mathrm{H})$ lower 8.2. This lack does not mean that there are no low-metallicity $\mathrm{H}_{\text {II }}$ regions in the outer parts of spiral galaxies. Rather, it is caused by the following reason: the relationship between oxygen abundance and strong line intensities is double-valued with two distinctive parts usually known as the lower and upper branches of the $R_{23}-\mathrm{O} / \mathrm{H}$ 


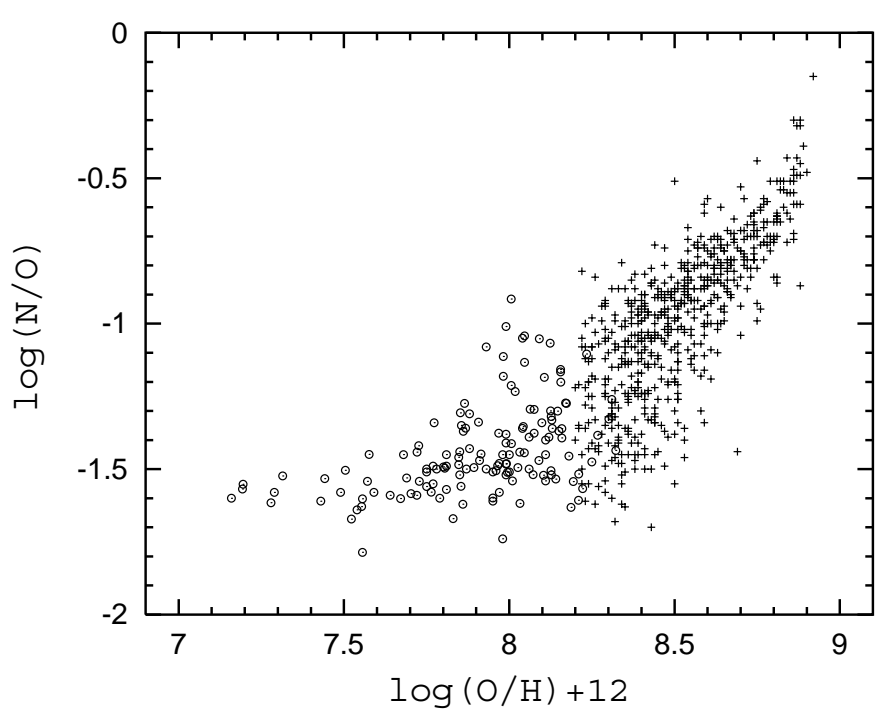

Fig. 4. The N/O-O/H for $\mathrm{H}_{\mathrm{II}}$ regions in spiral (pluses) and irregular (circles) galaxies.

diagram. Thus, one has to know in advance on which of the two branches the $\mathrm{H}$ II region lies. The above expression for the oxygen abundance determination in H II regions, Eq. (9), is only valid for $\mathrm{H}_{\text {II }}$ regions which belong to the upper branch, with $12+\log (\mathrm{O} / \mathrm{H})$ higher than around 8.2. It has been known for a long time (Searle 1971; Smith 1975) that disks of spiral galaxies can show radial oxygen abundance gradients, in the sense that the oxygen abundance is higher in the central part of the disk and decreases with galactocentric distance. Thus, we start from the $\mathrm{H}$ II regions in the central part of disk and move outward until the radius $R^{*}$ where the oxygen abundance decreases to around $12+\log (\mathrm{O} / \mathrm{H})=8.2$. An unjustified use of Eq. (9) in the determination of the oxygen abundance in low-metallicity $\mathrm{H}$ II regions beyond $R^{*}$ would result in overestimated oxygen abundances, and would cause a false bend in the slope of abundance gradients to appear. Therefore, $\mathrm{H}$ II regions with galactocentric distances larger than $R^{*}$, those with $12+\log (\mathrm{O} / \mathrm{H})$ less than 8.2 were rejected.

The general N/O-O/H diagram for $\mathrm{H}$ II regions in spiral and irregular galaxies is shown in Fig. 4.

\section{The nitrogen production sites traced by the observational data}

\subsection{The $\mathrm{N} / \mathrm{O}-\mathrm{O} / \mathrm{H}$ diagram}

The observed trend of nitrogen abundance with metallicity as measured by oxygen abundance has been widely used as a clue to identify the sites for nitrogen production (Edmunds \& Pagel 1978; Garnett 1990; Pilyugin 1992, 1993; Marconi et al. 1994; Thuan et al. 1995; Izotov \& Thuan 1999; Henry et al. 2000; among others). Conclusions about the nitrogen production sites are reached from the interpretation of three prominent features in the observed $\mathrm{N} / \mathrm{O}-\mathrm{O} / \mathrm{H}$ diagram $: i$ ) the remarkable constancy of N/O in the lowest metallicity $(12+\log (\mathrm{O} / \mathrm{H})<$ 7.6) galaxies, and the fact that, up to $\mathrm{O}$ abundances around $12+\log (\mathrm{O} / \mathrm{H})=8.3$ the lower envelope of $\mathrm{N} / \mathrm{O}$ is equal to the $\mathrm{N} / \mathrm{O}$ value at low $\mathrm{O}$ abundances; ii) beginning at roughly $12+\log (\mathrm{O} / \mathrm{H})=7.6$, there is a large scatter in $\mathrm{N} / \mathrm{O}$ at a given $\mathrm{O} / \mathrm{H}$ value; iii) beginning at roughly $12+\log (\mathrm{O} / \mathrm{H})=8.3$, the lower envelope of $\mathrm{N} / \mathrm{O}$ increases with $\mathrm{O} / \mathrm{H}$.

In the early 1990 s, several studies were devoted to the explanation of the N/O-O/H diagram (Garnett 1990; Pilyugin 1992, 1993; Vila-Costas \& Edmunds 1992, Marconi et al. 1994). The problem of the scatter in $\mathrm{N} / \mathrm{O}$ at a given $\mathrm{O} / \mathrm{H}$ value has attracted particular attention. Two mechanisms have been suggested to explain the scatter. The first mechanism is timedelayed production of nitrogen. The second mechanism is selective heavy-element loss through enriched galactic winds. As mentioned above, the observed present-day N/O-O/H diagram at low metallicities differs significantly from the earlier one: while the earlier diagram suggests that the scatter in N/O grows as $\mathrm{O} / \mathrm{H}$ decreases, the present one shows exactly the reverse. Therefore the interpretation suggested in the early 1990s has been revised in later studies.

Thuan et al. (1995) and Izotov \& Thuan (1999) have concluded that the small dispersion of N/O at low metallicities is strong evidence against any time-delayed production of nitrogen in the lowest metallicity blue compact galaxies and the constancy of N/O can be explained only by nitrogen production in short-lived massive stars, i.e. oxygen and nitrogen must be made in the same massive stars. Nitrogen production by massive stars was also suggested by Matteucci (1986). Thuan et al. (1995) and Izotov \& Thuan (1999) have concluded that the large dispersion of N/O in galaxies with $12+\log (\mathrm{O} / \mathrm{H})>7.6$ can be explained by the addition of nitrogen production in intermediate-mass stars; by the time intermediate-mass stars have evolved and released their nucleosynthetic products (100-500 Myr), all galaxies have become enriched to $7.6<12+\log (\mathrm{O} / \mathrm{H})<8.2-$ the delayed release of nitrogen greatly increase the scatter in N/O. Close examination of the $\mathrm{N} / \mathrm{O}-\mathrm{O} / \mathrm{H}$ diagram shows that this scenario for the history of galaxy enrichment in nitrogen meets difficulties in galaxies with $12+\log (\mathrm{O} / \mathrm{H})>7.6$. Indeed, the $\mathrm{N} / \mathrm{O}$ value in the Small Magellanic Cloud is close to that in the lowest metallicity blue compact galaxies, Fig. 1, (see below). According to the above scenario, only massive stars are responsible for the $\mathrm{N} / \mathrm{O}$ value in the Small Magellanic Cloud. Then one has to conclude that most of the stars in the Small Magellanic Cloud have formed recently so that the nitrogen-producing stars have not yet returned their nucleosynthesis products to the interstellar medium because they have not had enough time to evolve. In that scenario, most of the stars in the Small Magellanic Cloud formed in last $100-500 \mathrm{Myr}$, which is not the case.

Henry et al. (2000) have concluded that the constancy of the N/O in metal-poor galaxies is entirely and naturally explained if they are characterized by historically low star formation rates, with nearly all of the nitrogen being produced by intermediate mass stars between 4 and $8 M_{\odot}$, with a moderate time delay (a characteristic lag time of roughly $250 \mathrm{Myr}$ following their formation). Those authors have found that this moderate time delay in nitrogen release by intermediate-mass stars does not appear to be an important factor in the evolution of this element. They concluded that the scatter is caused by intermittent increases in nitrogen caused by local contamination by Wolf-Rayet stars or luminous blue variables. Given that the 


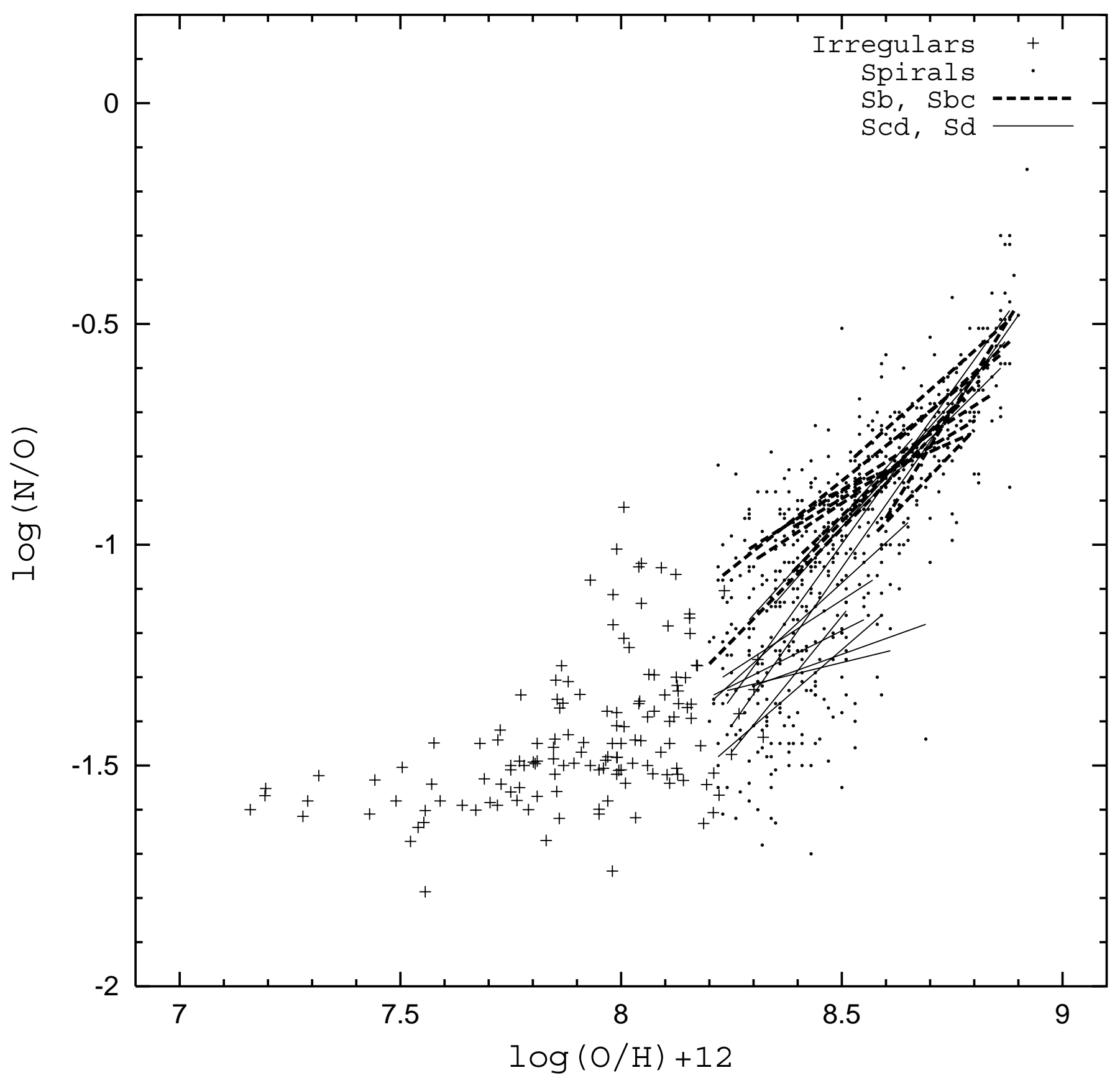

Fig. 5. The $\mathrm{N} / \mathrm{O}-\mathrm{O} / \mathrm{H}$ diagram. The pluses are $\mathrm{H}$ II regions in irregular galaxies. The points are $\mathrm{H}$ II regions in spiral galaxies. The thick dashed lines are the $\mathrm{N} / \mathrm{O}-\mathrm{O} / \mathrm{H}$ trends in $\mathrm{Sb}$ and Sbc galaxies (T-type $=3,4$ ). The thin solid lines are the $\mathrm{N} / \mathrm{O}-\mathrm{O} / \mathrm{H}$ trends in $\mathrm{Scd}$ and $\mathrm{Sd}$ galaxies (T-type $=6,7)$.

scatter is large (more than $0.3 \mathrm{dex}$ at $12+\log (\mathrm{O} / \mathrm{H})=7.9)$, this conclusion appears to be in conflict with their other conclusion that nearly all of the nitrogen is produced by intermediate-mass stars between 4 and $8 M_{\odot}$.

Thus, the existing explanations of the large scatter in N/O at a given $\mathrm{O} / \mathrm{H}$ do not appear to be satisfactory. We have found that the low-metallicity $\mathrm{H}_{\text {II }}$ regions in the outer parts of spiral galaxies are more suitable objects for establishing the origin of the scatter than $\mathrm{H}$ II regions in irregular galaxies. Our finding is the following. The positions of spiral galaxies of different morphological types in the $\mathrm{N} / \mathrm{O}-\mathrm{O} / \mathrm{H}$ diagram are shown in Fig. 5 by different symbols. The dashed lines correspond to $\mathrm{Sb}$ and $\mathrm{Sbc}$ galaxies (T-type $=3,4$ ), and the solid lines to Scd and Sd galaxies (T-type $=6,7$ ). Figure 5 shows clearly that the N/O values in $\mathrm{H}$ II regions of galaxies of early morphological types are systematically higher than the N/O values in $\mathrm{H}$ II regions with the same $\mathrm{O} / \mathrm{H}$ value in galaxies of late morphological types. It can be also seen in Fig. 6 where the N/O ratio at $12+\log (\mathrm{O} / \mathrm{H})=8.2$ is shown as a function of numerical morphological T-type. It is interesting to note that although Vila-Costas \& Edmunds (1992) have concluded that there is no obvious difference between morphological types, a hint that 


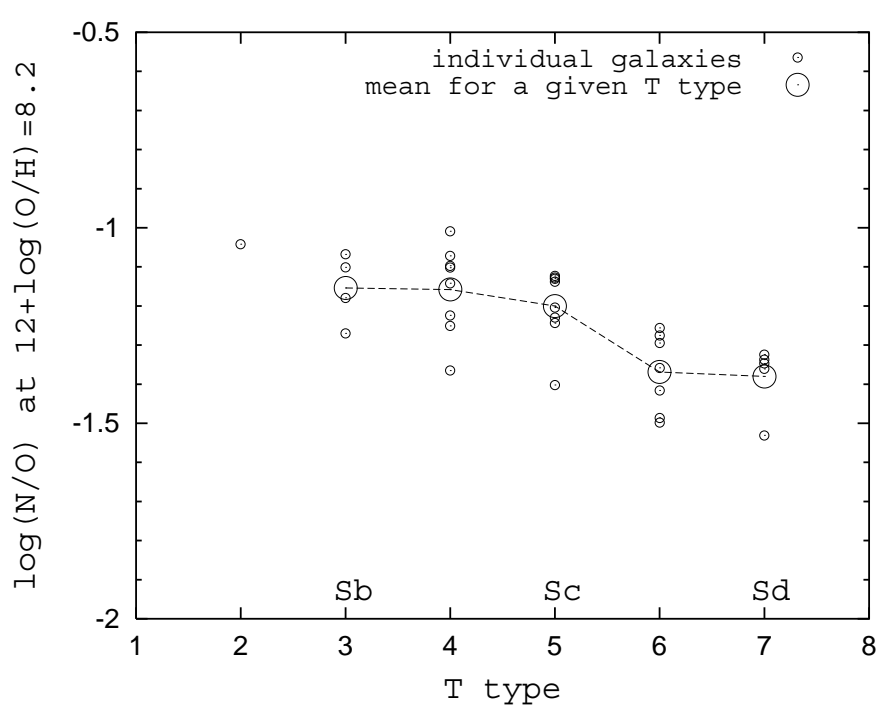

Fig. 6. The small circles are the $\log (\mathrm{N} / \mathrm{O})$ values at $12+\log (\mathrm{O} / \mathrm{H})=$ 8.2 in individual galaxies as a function of the T-type. The large circles are the $\log (\mathrm{N} / \mathrm{O})$ values at $12+\log (\mathrm{O} / \mathrm{H})=8.2$ averaged over all galaxies of the same T-type.

the N/O ratios in the $\mathrm{H}$ II regions of the late-type spiral galaxies are lower than the ones in the $\mathrm{H}$ II regions with the same oxygen abundance in early-type spiral galaxies can be seen in their Fig. 5. The much more distinct correlation of the N/O ratio with galaxy morphological type in the present study is due to the fact that the positions of $\mathrm{H}$ in regions in the $\mathrm{N} / \mathrm{O}-\mathrm{O} / \mathrm{H}$ diagram derived here with the $P$-method are more accurate than those derived by Vila-Costas \& Edmunds (1992).

It is well-known that galaxies of different morphological types have different star formation histories. The schematic star formation histories of galaxies of different morphological types according to Sandage (1986) are presented in Fig. 7. The star formation rate is in arbitrary units. Figure 7 shows that the spiral galaxies with early morphological types have a significantly larger fraction of old stars (with ages 8-13 Gyr) than the galaxies of late morphological types. This suggests that the high $\mathrm{N} / \mathrm{O}$ values in the galaxies of early morphological types can be caused by the contribution of low-mass stars (with masses around a solar mass) to the nitrogen production, while in the galaxies of late morphological types these stars have not yet returned their nucleosynthesis products to the interstellar medium because they have not had enough time to evolve. The long time delay for nitrogen production appears to be an important factor in the evolution of this element. The scatter in $\mathrm{N} / \mathrm{O}$ values at a given $\mathrm{O} / \mathrm{H}$ can be naturally explained by differences in star formation histories in galaxies. This conclusion is not new. More than twenty years ago, Edmunds \& Pagel (1978) have suggested that observations of the N/O abundance ratio in external galaxies can be understood if nitrogen is manufactured principally in stars of $1-2.5 M_{\odot}$. The N/O ratio of a galaxy then becomes an indicator of the time that has elapsed since the bulk of star formation occurred, or in other words of the nominal "age" of the galaxy.

The explanation that the increase of the lower envelope of $\mathrm{N} / \mathrm{O}$ with $\mathrm{O} / \mathrm{H}$, beginning roughly at $12+\log (\mathrm{O} / \mathrm{H})=8.3$, is

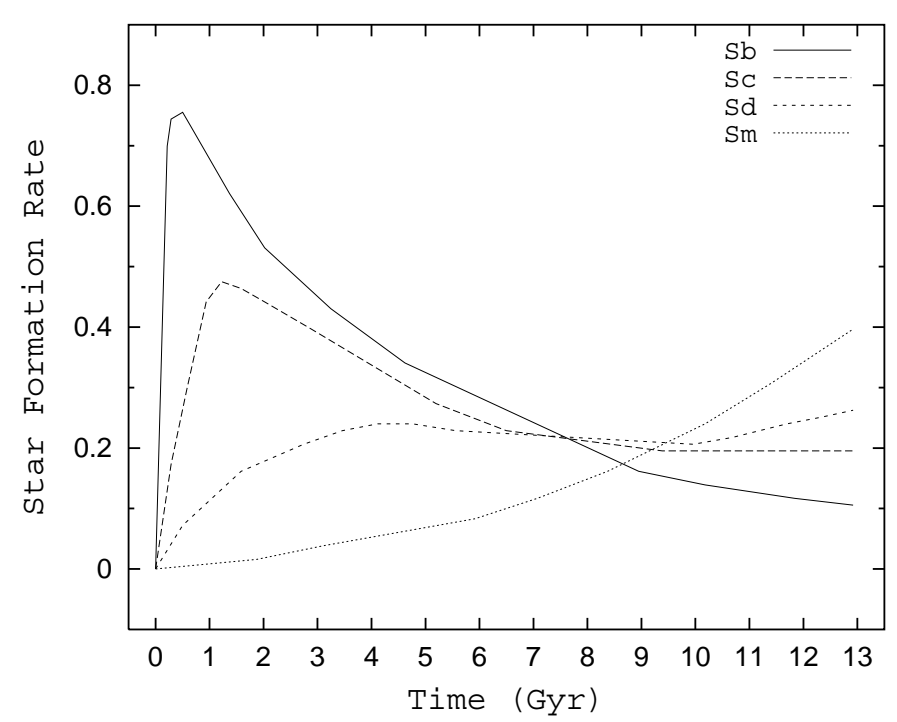

Fig. 7. The schematic star formation history of galaxies of different morphological types according to Sandage (1986). The star formation rate is in arbitrary units.

controlled by the metallicity-dependent nitrogen production in intermediate-mass stars is commonly accepted now.

Thus, the present and previous studies of $\mathrm{N} / \mathrm{O}-\mathrm{O} / \mathrm{H}$ diagram lead to the following conclusions about the sites of nitrogen production: (1) at low metallicities the metallicityindependent nitrogen production takes place in short-lived massive stars with no time delay between the release of nitrogen and oxygen or/and in intermediate-mass stars with moderate time delay, with a characteristic time delay of a few hundred Myr between the release of nitrogen and oxygen; (2) at metallicities higher than roughly $12+\log (\mathrm{O} / \mathrm{H})=8.3$, the metallicity-dependent nitrogen production by intermediatemass stars dominates; (3) the contribution of low-mass stars to the nitrogen production with long time delay, with characteristic time delay of several Gyr, can be significant.

Observational data of other kinds can be used to verify and refine conclusions reached by us and other authors from consideration of the $\mathrm{N} / \mathrm{O}-\mathrm{O} / \mathrm{H}$ diagram.

\subsection{The self-enriched $\mathrm{H}$ II regions}

Generally, stars of different masses from all the previous star formation events can make contributions to the $\mathrm{N}$ abundances measured in the interstellar medium. Fortunately, there are cases where the contribution to nitrogen production from massive stars from a current star formation event can be established. Kunth \& Sargent (1986) have suggested that H II regions may "pollute" themselves on short $\left(10^{6} \mathrm{yr}\right)$ timescales with nucleosynthetic products from the current burst of star formation. This suggestion is not beyond question. There may be some time lag $\left(\sim 10^{7} \mathrm{yrs}\right)$ between supernova explosions and the appearance of the freshly produced oxygen in the warm phase of the interstellar gas (Tenorio-Tagle 1996; Kobulnicky \& Skillman 1996, 1997). There are however examples where the enrichment in heavy elements by massive stars on short 
timescales is exhibited. Those examples can tell us something about the nitrogen production by massive stars.

Let us test the conclusion of Izotov \& Thuan (1999) that the nitrogen in low-metallicity (with $12+\log (\mathrm{O} / \mathrm{H})<7.6$ ) blue compact galaxies is produced by massive stars only. The best fit to Izotov \& Thuan's data results in $\Delta \mathrm{N} / \Delta \mathrm{O} \sim 1 / 40$ (solid line in Fig. 8a).

Kobulnicky \& Skillman (1996) carried out an investigation of the multiple starburst knots in the nucleus of the lowmetallicity irregular galaxy NGC 4214. From optical spectroscopy at distinct spatial locations they reveal large scale (about 200 pc) variations in the oxygen abundance. The southernmost, and apparently youngest emission line region exhibits a higher oxygen abundance than the rest of the galaxy. The $\mathrm{N} / \mathrm{O}$ ratio is lower in this region. Kobulnicky \& Skillman suggest that recent oxygen polution from supernovae has occurred in this very young population of massive stars. Their data (taken from their Fig. 8) are shown in Fig. 8b by triangles. The variation of oxygen and nitrogen abundances computed with $\Delta \mathrm{N}=\frac{1}{40} \Delta \mathrm{O}$ is shown by the solid line. Examination of Fig. $8 \mathrm{~b}$ shows that Kobulnicky and Skillman's data agree satisfactory with the prediction of the model with $\Delta \mathrm{N}=\frac{1}{40} \Delta \mathrm{O}$ (solid line), however these data agree equally satisfactory with the prediction of the model without nitrogen production by massive stars, $\Delta \mathrm{N}=0$, (dashed line).

Cunha \& Lambert $(1992,1994)$ have measured the N, C, O, $\mathrm{Si}$, and $\mathrm{Fe}$ abundances of main-sequence $\mathrm{B}$ stars in the Orion star formation region. There is a time lag of about $11 \mathrm{Myr}$ between the formation of the oldest and the youngest subgroups of stars in the Orion association. The stars in the youngest subgroup are enriched in heavy elements relative to the stars belonging to the oldest subgroup, i.e. the later subgeneration of stars have formed out of material whose chemical composition has been altered by the nucleosynthesis products of massive stars from previous subgenerations. Again, the track with $\Delta \mathrm{N} / \Delta \mathrm{O} \sim 1 / 40$ (solid line) and the track with $\Delta \mathrm{N}=0$ (dashed line) cannot be chosen on the basis of observational data (Fig. 8c).

Thus, the consideration of the $\mathrm{H}_{\mathrm{II}}$ regions taken to be selfenriched does not result in a firm argument pro or contra nitrogen production by massive stars.

\subsection{The planetary nebulae}

It is usually accepted that the oxygen abundances measured in planetary nebulae reflect the initial abundance of the progenitor stars while the nitrogen abundances in planetary nebulae with progenitors from some range of stellar mass are modified by the progenitor's evolution. Then the oxygen abundance in the planetary nebula can serve as some kind of "age" indicator of the progenitor if the chemical evolution of the galaxy (the history of galaxy enrichment in oxygen) is established. It should be stressed that we do not imply to derive the age of the progenitor of individual planetary nebula based on its oxygen abundance. Indeed, the temporal evolution of the oxygen abundance in our Galaxy is nonsmooth (Pilyugin \& Edmunds 1996a,b), therefore the oxygen abundance in the individual
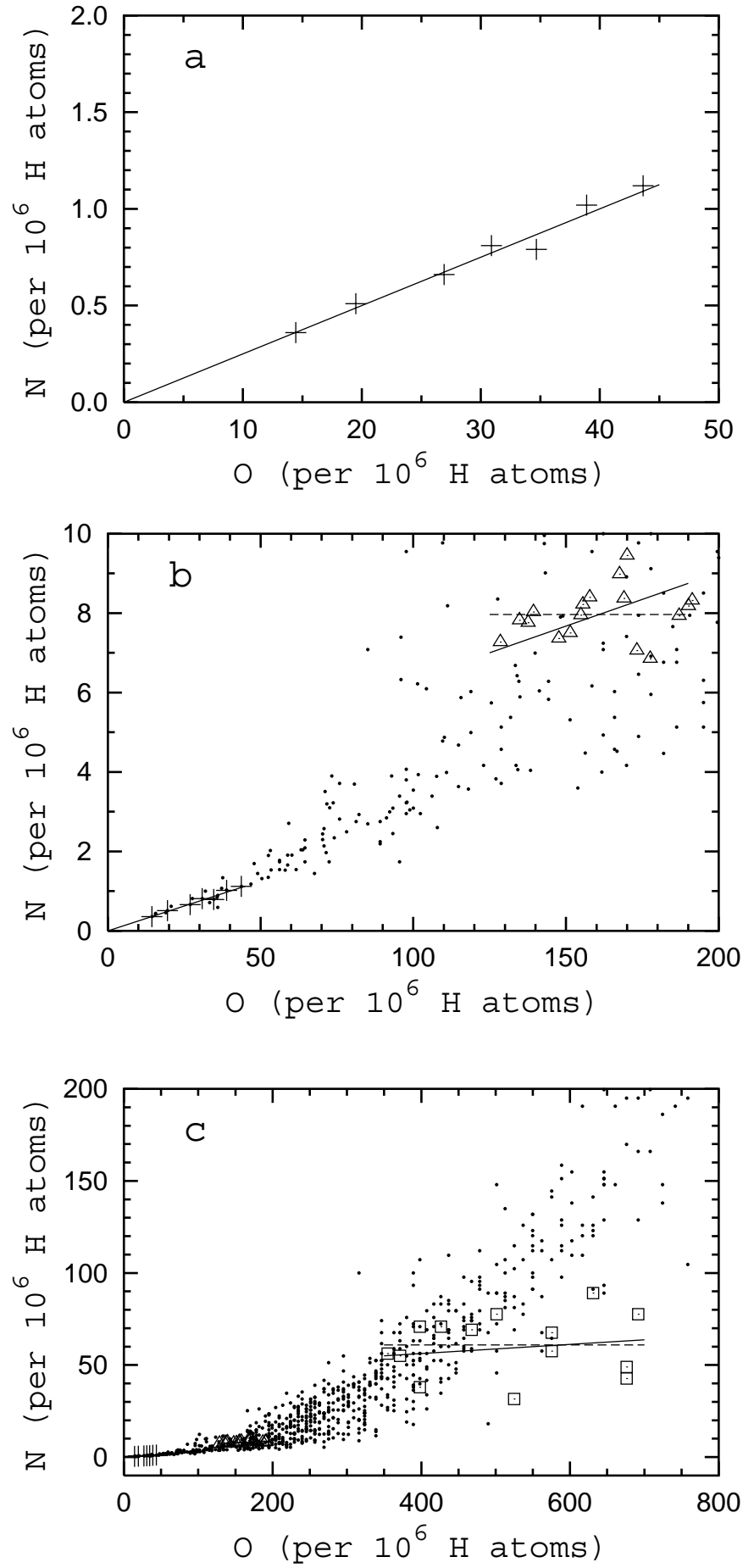

Fig. 8. Examples in which the enrichment in heavy elements by massive stars is expected. a). Pluses are $\mathrm{H}_{\text {II }}$ regions in extremely metalpoor blue compact dwarf galaxies from Izotov \& Thuan (1999). The solid line is the best fit to them. b). Triangles are distinct spatial locations of multiple starburst knots within the galaxy NGC 4214 (Kobulnicky \& Skillman 1996). Points are the same objects as in Fig. 4. The solid line is the track corresponding to the ratio of nitrogen to oxygen enrichment yields $\Delta \mathrm{N}=\frac{1}{40} \Delta \mathrm{O}$. The dashed line corresponds to $\Delta \mathrm{N}=0$. c). Squares are $\mathrm{B}$ stars from the Orion association (Cunha \& Lambert 1994). Points are the same objects as in Fig. 1. The solid line is the track corresponding to a ratio of nitrogen to oxygen enrichment yields $\Delta \mathrm{N}=\frac{1}{40} \Delta \mathrm{O}$. The dashed line corresponds to $\Delta \mathrm{N}=0$. 


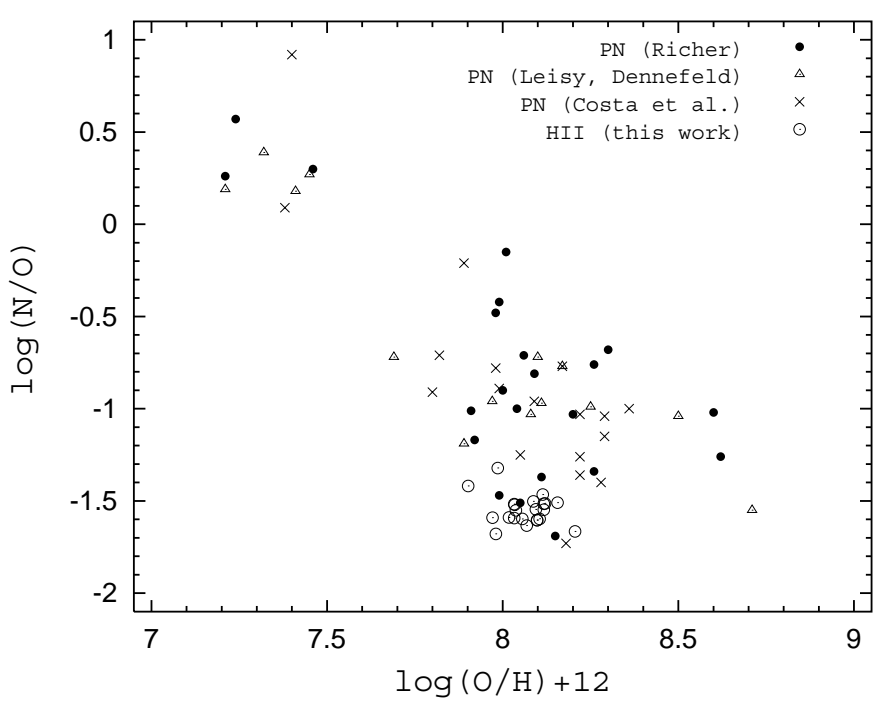

Fig. 9. The $\mathrm{O} / \mathrm{H}-\mathrm{N} / \mathrm{O}$ diagram for $\mathrm{H}$ II regions and planetary nebulae in the Small Magellanic Cloud. The points are original data for planetary nebulae from Richer (1993), the triangles are corrected data for planetary nebulae from Leisy \& Dennefeld (1996), the crosses are "corrected" data for planetary nebulae from Costa et al. (2000). Open circles are data for $\mathrm{H}_{\text {II }}$ regions from Table 1.

planetary nebula is not a reliable "age" indicator of its progenitor. We only mean that the assumption, that the oxygen-rich planetary nebulae (with $12+\log \mathrm{O} / \mathrm{H}>7.7$ in the case of the Small Magellanic Cloud) were formed from younger (in average) and therefore more massive stars than the oxygen-poor planetary nebulae (with $12+\log \mathrm{O} / \mathrm{H}<7.5$ in the case of the Small Magellanic Cloud), is justified. Then the examination of nitrogen abundances of planetary nebulae with different oxygen abundances allows to make conclusion about the contribution of the intermediate- and low-mass stars to the enrichment of the interstellar medium in nitrogen.

The determinations of the chemical composition in a number of planetary nebulae of the Small Magellanic Cloud are available now (Richer 1993; Leisy \& Dennefeld 1996; Costa et al. 2000). This data allows to make conclusion about the contribution of the intermediate- and low-mass stars to the enrichment of the interstellar medium in nitrogen. Richer (1993) reported the oxygen and nitrogen abundances for twenty-five planetary nebulae in the Small Magellanic Cloud, Leisy \& Dennefeld (1996) for fifteen planetary nebulae, and Costa et al. (2000) for twenty-one planetary nebulae

The list of Richer (1993) has ten planetary nebulae in common with the list of Leisy \& Dennefeld (1996) and fifteen planetary nebulae in common with the list of Costa et al. (2000). Comparison of the oxygen abundances in common planetary nebulae shows that the ones derived by Costa et al. (2000) and Leisy \& Dennefeld (1996) are shifted towards higher oxygen abundances when compared to those derived by Richer (1993). In order to have a homogeneous set of abundances, the abundances of both oxygen and nitrogen of Costa et al. and of Leisy $\&$ Dennefeld were reduced to the system of Richer. We chose Richer's system because his planetary nebulae oxygen abundances are in better agreement with those in $\mathrm{H}$ II regions.

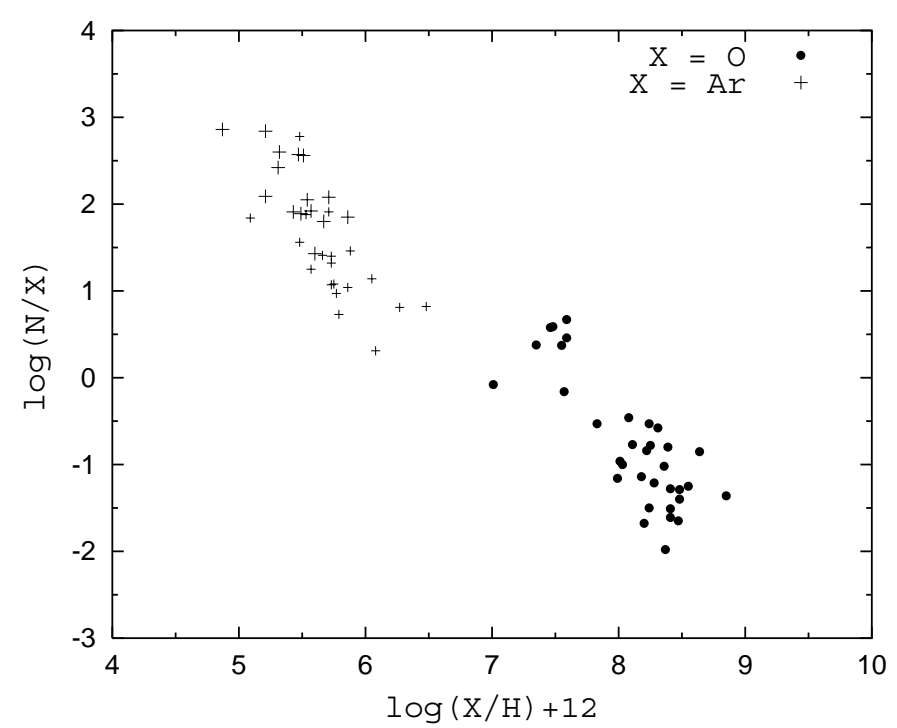

Fig. 10. The $\mathrm{N} / \mathrm{O}-\mathrm{O} / \mathrm{H}$ diagram (points) and $\mathrm{N} / \mathrm{Ar}-\mathrm{Ar} / \mathrm{H}$ diagram (pluses) for planetary nebulae in the Small Magellanic Cloud. The original data from Leisy \& Dennefeld (1996) and Costa et al. (2000) are shown.

The $\mathrm{O} / \mathrm{H}-\mathrm{N} / \mathrm{O}$ diagram for $\mathrm{H}$ II regions and planetary nebulae in the Small Magellanic Cloud is shown in Fig. 9. The points are original data for planetary nebulae from Richer (1993), the triangles are the corrected data for planetary nebulae from Leisy \& Dennefeld (1996), the crosses are the corrected data for planetary nebulae from Costa et al. (2000). The open circles are data for $\mathrm{H}$ II regions from Table 1. Inspection of Fig. 9 shows that the scatter of oxygen abundances in planetary nebulae of the Small Magellanic Cloud is large, more than one order of magnitude. An anti-correlation between N/O and $\mathrm{O} / \mathrm{H}$ is clearly seen. The most evident explanation of the scatter is that the oxygen abundance of planetary nebulae traces the time of the progenitor star formation, the oxygen-poor planetary nebulae progenitors being older (Leisy \& Dennefeld 1996; Costa et al. 2000).

Other possible origins of the oxygen abundance scatter and the $\mathrm{N} / \mathrm{O}-\mathrm{O} / \mathrm{H}$ trend have also been discussed in the literature. Leisy \& Dennefeld (1996, 2000) considered the possibility that the oxygen abundance in planetary nebulae does not reflect the initial composition of the progenitor star, that it can be destroyed in Type I planetary nebulae (He-N rich objects, Peimbert 1978) as well as produced in non-Type I planetary nebulae, during the progenitor star life-time. If this is the major reason for the oxygen abundance scatter and the N/O$\mathrm{O} / \mathrm{H}$ trend then: $i$ ) the $\mathrm{Ar} / \mathrm{O}$ ratio would be higher in planetary nebulae with low $\mathrm{O}$ abundances and $\mathrm{ii}$ ) the $\mathrm{N} / \mathrm{Ar}-\mathrm{Ar} / \mathrm{H}$ diagram would differ radically from the N/O-O/H diagram since $\mathrm{Ar}$ is not affected by the evolution of intermediate-mass stars. Milingo et al. (2002) have undertaken a large spectroscopic survey of Galactic planetary nebulae with the goal of providing a homogeneous spectroscopic database, as well as a set of consistently determined abundances, especially for oxygen, sulfur, chlorine, and argon. Their data (their Fig. 2) do not show a tendency for $\mathrm{Ar} / \mathrm{O}$ to decrease with $\mathrm{O} / \mathrm{H}$. They found an average $\mathrm{Ar} / \mathrm{O}=0.0051 \pm 0.0020$ for planetary nebulae, in agreement 
with $\mathrm{Ar} / \mathrm{O}=0.0055$ derived by Izotov \& Thuan (1999) for H II regions in blue compact dwarf galaxies.

Figure 10 shows the $\mathrm{N} / \mathrm{O}-\mathrm{O} / \mathrm{H}$ diagram (points) together with the N/Ar-Ar/H diagram (pluses) for planetary nebulae in the Small Magellanic Cloud. The original data from Leisy \& Dennefeld (1996) and Costa et al. (2000) are shown. There is both a scatter in argon abundances and an anti-correlation between N/Ar and $\mathrm{Ar} / \mathrm{H}$. Given that $\mathrm{Ar} / \mathrm{O}$ is independent of $\mathrm{O} / \mathrm{H}$ and that the N/Ar-Ar/H diagram is similar to the N/O-O/H diagram, one can conclude that possible oxygen production and/or destruction during the progenitor star life-time do not dramatically change the oxygen abundance of the progenitor star, and oxygen can be used as a metallicity tracer, at least in the first approximation.

The bulk of planetary nebulae in the Small Magellanic Cloud has oxygen abundances compatible with those in $\mathrm{H}$ II regions (Fig. 9), a fact already mentioned by different authors. Based on their model for the chemical evolution of the Small Magellanic Cloud, Costa et al. (2000) have concluded that the progenitors of these planetary nebulae have been formed within the last 1-2 Gyr. They also estimated an age of about $12 \mathrm{Gyr}$ for the progenitor of the most oxygen-poor planetary nebula in the Small Magellanic Cloud.

Thus, the oxygen abundances measured in planetary nebulae reflect the initial abundance of the progenitor stars while the nitrogen abundances are modified by the progenitors' evolution. If the initial nitrogen abundance of the progenitor star is known, then the difference between the amount of nitrogen in the ejected matter (i.e. in the planetary nebula) and the initial amount of nitrogen in this matter is the amount of nitrogen manufactured by the star. The initial nitrogen abundance in the progenitor star can be estimated in the following way. Examination of Table 1 shows that the variation in the $\mathrm{N} / \mathrm{O}$ ratio from $\mathrm{H}_{\text {II }}$ region to $\mathrm{H}_{\mathrm{II}}$ region in the Small Magellanic Cloud is small within the errors in abundance determinations. Thus we can adopt this N/O ratio corresponds as the "equilibrium" ratio in the interstellar medium of the Small Magellanic Cloud at the present-day epoch. It is unlikely that this value is increased or decreased by the same amount of local self-enrichment in nitrogen or oxygen in all HII regions. As shown above, the equilibrium N/O ratio in the interstellar medium of a galaxy can be increased by the contribution of low-mass stars to the nitrogen production. The N/O ratio in the $\mathrm{H}_{\text {II }}$ regions of the Small Magellanic Cloud is very close to that in the $\mathrm{H}$ II regions of the most oxygen-poor blue compact galaxies, Fig. 1, i.e. the present-day equilibrium N/O ratio in the interstellar medium of the Small Magellanic Cloud corresponds to the minimum value of the N/O ratio. It has not changed up to now, and therefore can be adopted as the initial N/O ratio for the progenitor star of any age. Thus, the initial nitrogen abundance in the progenitor star can be estimated from the oxygen abundance measured in the planetary nebula (since this oxygen abundance reflects the initial oxygen abundance of the progenitor star) and from the $\mathrm{N} / \mathrm{O}$ ratio measured in $\mathrm{H}$ in regions. The amount of nitrogen manufactured in the star can then be estimated by subtracting the initial nitrogen abundance from the amount of nitrogen observed in the planetary nebula. The contribution of low mass stars to the nitrogen production can then be compared to that of the intermediate mass stars, as shown below.

Galactic Type I (nitrogen-rich) planetary nebulae are believed to correspond to the high mass end of the stellar progenitors of planetary nebulae (Torres-Peimbert \& Peimbert 1997, and references therein). The planetary nebulae in the Small Magellanic Cloud do not appear to fit in this scheme. Figure 9 shows that nitrogen abundances in oxygen-poor planetary nebulae are higher by a factor 3-5 than that in oxygen-rich ones. Since the oxygen-poor planetary nebulae in the Small Magellanic Cloud seem to be formed from older and therefore less massive stars, then Type I planetary nebulae progenitors can be less massive than expected. Leisy \& Dennefeld (1996) have concluded that, with their definition based only on $\mathrm{N}$ and He abundances, Type I planetary nebulae do not form an homogeneous class.

Let us consider the Milky Way planetary nebulae. In contrast to the Small Magellanic Cloud, the Milky Way shows both an oxygen and nitrogen radial abundance gradient. In order to exclude initial chemical composition differences of the planetary nebula progenitors caused by the radial abundance gradient, only planetary nebulae in the solar vicinity, with galactocentric distances between 7.5 and $9.5 \mathrm{kpc}$, will be considered. Abundances in large samples of Galactic planetary nebulae have been determined by Aller \& Czyzak (1983), Aller \& Keyes (1987), Köppen et al. (1991), and Kingsburgh $\&$ Barlow (1994). The positions of the Galactic solar vicinity planetary nebulae in the $\mathrm{N} / \mathrm{O}-\mathrm{O} / \mathrm{H}$ diagram are shown in Fig. 11 by points. The galactocentric distances were obtained using the distances to planetary nebulae from Cahn et al. (1992). When the distance is not given by Cahn et al., it is taken from Kingsburgh \& English (1992) or from Maciel (1984). The open circle in Fig. 11 corresponds to the oxygen and nitrogen abundances at the solar galactocentric distance as traced by the H II regions (Pilyugin et al. 2002). The planetary nebulae in the Small Magellanic Cloud are shown by pluses in Fig. 11, the open square corresponds to the average $\mathrm{N} / \mathrm{O}$ and $\mathrm{O} / \mathrm{H}$ values in the $\mathrm{H}_{\text {II }}$ regions of the Small Magellanic Cloud.

Inspection of Fig. 11 shows that the bulk of Galactic planetary nebulae have oxygen abundances in a relatively narrow range. These planetary nebulae are believed to be relatively "young", i.e. they correspond to the intermediate-mass stellar progenitors of planetary nebulae The difference between nitrogen abundances in these planetary nebulae and in $\mathrm{H}$ in regions (this difference reflects the contribution of freshly produced nitrogen) is higher than the corresponding value for the "young" planetary nebulae of the Small Magellanic Cloud. It is not surprising and confirms the widely accepted point of view that the increase of the lower envelope of $\mathrm{N} / \mathrm{O}$ with $\mathrm{O} / \mathrm{H}$, beginning at roughly at $12+\log (\mathrm{O} / \mathrm{H})=8.3$, is explained by the fact that the amount of nitrogen manufactured in the intermediate-mass stars increases with metallicity.

A few Galactic planetary nebulae have low oxygen abundances. If the oxygen abundance of planetary nebula traces the time of the progenitor star formation then the oxygen-poor Galactic planetary nebulae progenitors are old low-mass stars. However, the possibility that the oxygen-poor Galactic planetary nebulae were formed from matter captured by our Galaxy 


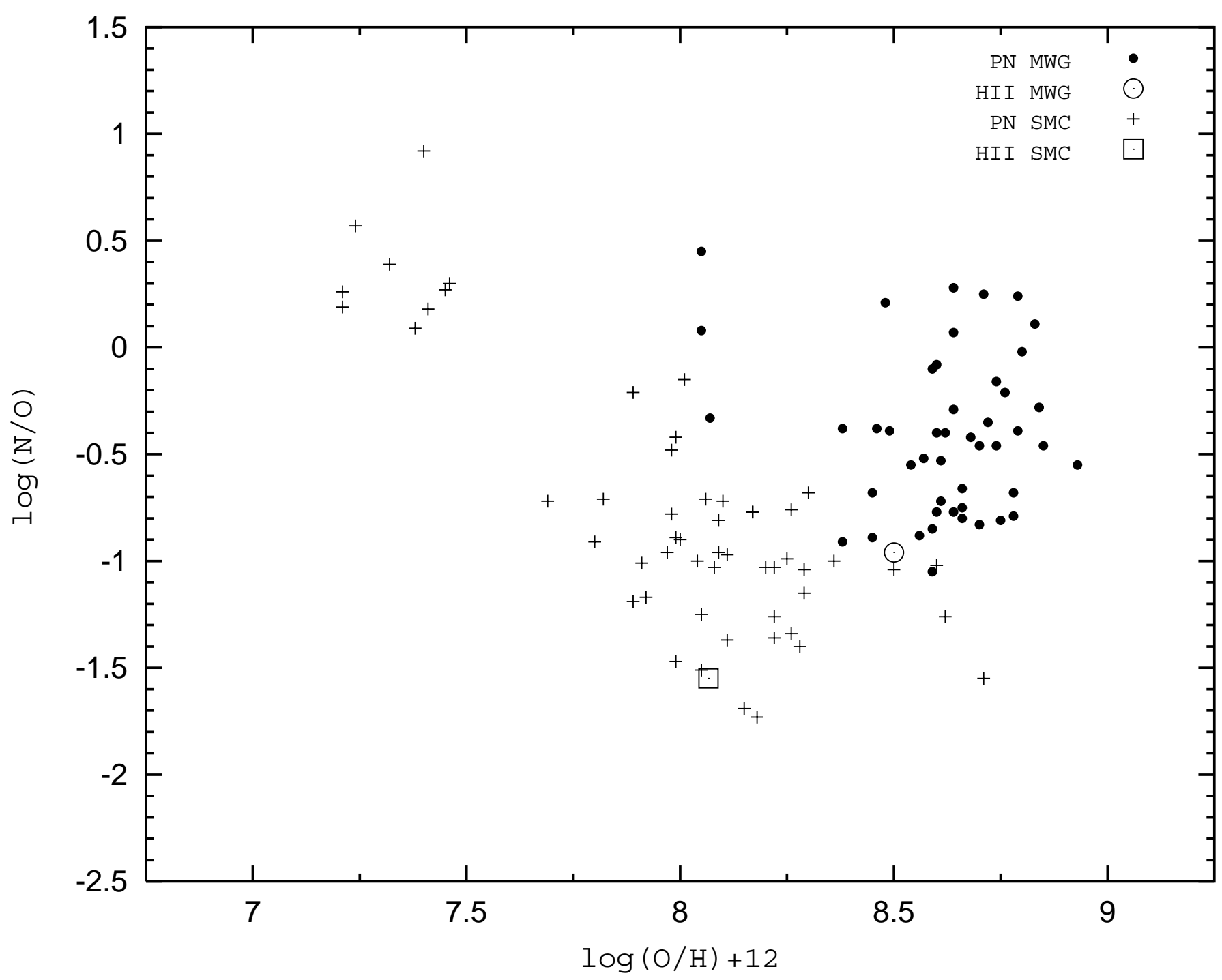

Fig. 11. The O/H-N/O diagram for the planetary nebulae in the Milky Way with galactocentric distances between 7.5 and $9.5 \mathrm{kpc}$ (points) and in the Small Magellanic Cloud (pluses). The open circle corresponds to the N/O and O/H at the solar galactocentric distance (8.5 kpc) as traced by $\mathrm{H}$ II regions. The open square corresponds to the average $\mathrm{N} / \mathrm{O}$ and $\mathrm{O} / \mathrm{H}$ values in the $\mathrm{H}$ II regions of the Small Magellanic Cloud.

from the Small Magellanic Cloud cannot be excluded. The Magellanic Stream is an excellent example of such a presentday capture. In this case the oxygen-poor Galactic planetary nebulae are "pseudo-old", i.e. they can also correspond to the intermediate-mass stellar progenitors of planetary nebulae like the oxygen-rich planetary nebulae. Although such a possibility cannot be excluded, for further discussion, we shall consider that the oxygen-poor Galactic planetary nebulae are really "old", i.e their progenitors are old low-mass stars.

Comparison between the old planetary nebulae of the Small Magellanic Cloud and the Milky Way Galaxy shows that N/O in old planetary nebulae slightly decreases with metallicity in contrast to the young planetary nebulae which show clearly the tendency for $\mathrm{N} / \mathrm{O}$ to increase with $\mathrm{O} / \mathrm{H}$, Fig. 11. This suggests that the contribution of low-mass stars to the nitrogen production decreases with metallicity as compared to the contribution of intermediate-mass stars. Data on old planetary nebulae in the Milky Way Galaxy are few in number, therefore more and better data are needed to verify this suggestion.
The average nitrogen abundance in the old planetary nebulae of the Small Magellanic Cloud is around 40-60 N atoms per million $\mathrm{H}$ atoms, Fig. 9. Unfortunately, young planetary nebulae with comparably low oxygen abundances $(7.3<12+$ $\log (\mathrm{O} / \mathrm{H})<7.5)$ are not available, therefore a direct comparison of the contributions of low-mass and intermediate-mass stars to the nitrogen production for low metallicities is not possible. However, if we adopt the usual assumption that the amount of nitrogen manufactured by intermediate-mass stars is independent of metallicity in the low metallicity range, then the young planetary nebulae with oxygen abundances around $12+\log (\mathrm{O} / \mathrm{H})=8.1$ can be used for such a comparison. The average nitrogen abundance in the young planetary nebulae of the Small Magellanic Cloud is around $10-15 \mathrm{~N}$ atoms per million $\mathrm{H}$ atoms (Fig. 9). At the same time, the fraction of the material returned into the interstellar medium by the intermediate-mass stars $\left(2 M_{\odot} \div 8 M_{\odot}\right)$ of a single stellar population is comparable with the fraction returned by the low-mass stars $\left(<2 M_{\odot}\right)$. Thus, in the low metallicity range, the contribution of low-mass 
stars to the nitrogen production appears to be rather significant and may even exceed the contribution of the intermediate-mass stars. The comparison between young planetary nebulae in the Milky Way Galaxy and in the Small Magellanic Cloud shows that there is a tendency for an increase of the amount of nitrogen manufactured in the intermediate-mass stars with metallicity. If this tendency holds also in the very low metallicity range, then the contribution of low-mass stars to the nitrogen production exceeds significantly the contribution of the intermediatemass stars at very low metallicities.

In summary, consideration of planetary nebulae in the Small Magellanic Cloud and the Milky Way Galaxy has shown that the contribution of low-mass stars to the nitrogen production is appreciable, and that at low metallicities the contribution of low-mass stars can exceed the contribution of the intermediate-mass stars. This confirms our conclusion, reached from the consideration of the $\mathrm{N} / \mathrm{O}-\mathrm{O} / \mathrm{H}$ diagram for spiral galaxies of different morphological types, that there is a longtime-delayed contribution to the nitrogen production.

\section{Discussion}

The present and previous studies suggest that at low metallicities the nitrogen production can take place $i$ ) in short-lived massive stars with no time delay between the release of nitrogen and oxygen, $i i)$ in intermediate-mass stars with a moderate time delay of a few hundred Myr between the release of nitrogen and oxygen, iii) in low-mass stars with a long time delay of a several Gyr between the release of nitrogen and oxygen. It is difficult to differentiate the contributions of massive and intermediate-mass stars to the enrichment of interstellar medium in nitrogen on the base of available observational data. An attempt to directly estimate the nitrogen production by massive stars from observational data of self-enriched $\mathrm{H}$ in regions has not resulted in a firm conclusion. Then the lowest observed $\mathrm{N} / \mathrm{O}$ value in low-metallicity $\mathrm{H}$ II regions (where the contribution of the massive stars from current star formation burst to the gas enrichment in heavy elements could be dominant) can be used as an estimate of the nitrogen production by massive stars. The delayed-release hypothesis, i.e. the moderate-timedelayed production of nitrogen by intermediate mass stars, predicts that $\mathrm{N} / \mathrm{O}$ drops while $\mathrm{O} / \mathrm{H}$ increases as massive stars begin to die and eject oxygen into the interstellar medium. At the point when all the massive stars have died, the N/O value is minimum. In the case of a strong star formation burst in lowmetallicity environment, the minimum N/O value corresponds approximately (or exactly in the case of the very first star formation burst) to the ratio of $\mathrm{N}$ to $\mathrm{O}$ yields in massive stars.

If the conclusion of Izotov \& Thuan (1999) - that the nitrogen observed in low-metallicity $(12+\log (\mathrm{O} / \mathrm{H})<7.6)$ blue compact galaxies has been produced by massive stars only - is correct, then the $\mathrm{N} / \mathrm{O}$ ratio in these galaxies $(\log (\mathrm{N} / \mathrm{O})=-1.6)$ is a lower limit for the global N/O ratio. In that case, there is little room left for the nitrogen production by intermediatemass stars. However, the N/O ratio in the $\mathrm{H}_{\text {II }}$ regions of the Small Magellanic Cloud is very close to that in the $\mathrm{H}$ II regions of the most oxygen-poor blue compact galaxies. The bulk of the intermediate-mass stars of the Small Magellanic Cloud have certainly had enough time to evolve (Pagel \& Tautvaišiene 1998; Mighell et al. 1998; Rich et al. 2000). If the value of $\log (\mathrm{N} / \mathrm{O})=-1.6$ corresponds to the element production by massive stars, then the contribution of intermediate-mass stars to the N/O ratio in the Small Magellanic Cloud $(\log (\mathrm{N} / \mathrm{O})=$ -1.55 , Table 1$)$ is negligibly small. It is worth noting that while the evolutionary intermediate-mass star models predict usually a significant nitrogen production (Renzini \& Voli 1981; van den Hoek \& Groenewegen 1997; Marigo 1996, 1998, 2001), the non-rotating models of Meynet \& Maeder (2002a,b) show very small nitrogen yields by intermediate-mass stars in low-metallicity environment. We do not consider the very small nitrogen yields by non-rotating intermediate-mass stars of Meynet \& Maeder as undisputed argument in favor of negligible small contribution of intermediate-mass stars to the enrichment of interstellar gas in nitrogen in low-metallicity environment, we only would like to note that such possibility cannot be excluded on the base of the existing models.

A crucial argument in favor of a significant production of nitrogen by intermediate-mass stars would be the existence of systems with very low N/O ratios. Inspection of Fig. 1 shows that two objects from the list of Kobulnicky \& Skillman (1998), CG $1116+51$ and Tol 65, have values of $\log$ N/O appreciably lower than -1.6 . However, Izotov et al. (2001) have recently redetermined the abundances for Tol 65 (Izotov et al. 2001) and found $\log (\mathrm{N} / \mathrm{O})=-1.642 \pm 0.024$ for Tol 65 , similar to other low-metallicity blue compact galaxies. Van Zee et al. (1997, 1998a) have also found some galaxies with very low $\log (\mathrm{N} / \mathrm{O})$ $(<-1.7)$. Izotov \& Thuan (1999) have argued that these low values can be caused by systematic errors. The reality of the low nitrogen abundances obtained in damped Ly $\alpha$ systems (Pettini et al. 1995; Lu et al. 1998; Pettini et al. 2002; Prochaska et al. 2002) was questioned by Izotov \& Thuan (1999). Therefore we will not repeat the discussion of these objects here. Recall that the low-metallicity range of the observed N/O-O/H diagram for the $\mathrm{H}$ II regions of irregular and blue compact galaxies has appreciably changed over the last decade. Can one exclude the possibility that the disagreement between the observed N/O$\mathrm{O} / \mathrm{H}$ diagram for the $\mathrm{H}_{\mathrm{II}}$ regions of irregular and blue compact galaxies and the observed $\mathrm{N} / \mathrm{O}-\mathrm{O} / \mathrm{H}$ diagram for the damped Ly $\alpha$ systems disappear in the near future?

Contini et al. (2002) have discussed the chemical properties of a sample of UV-selected galaxies, thought to be in a special stage in their evolution, following a powerful starburst. Several galaxies in the list of Contini et al. (2002) have values of $\log (\mathrm{N} / \mathrm{O})$ lower than -1.6. Unfortunately, those objects fall in or close to the transition region of the $R_{23}-\mathrm{O} / \mathrm{H}$ diagram, causing a large uncertainty in their abundance determination. The oxygen abundances in two objects with low $\mathrm{N} / \mathrm{O}$ ratio (\#30 and \#46) reported by Contini et al. are lower than $12+\log (\mathrm{O} / \mathrm{H})=8.0$. Therefore these objects are expected to belong to the lower branch of the $R_{23}-\mathrm{O} / \mathrm{H}$ diagram, and their abundances can be derived through the $P$-method for the lower branch of the $R_{23}-\mathrm{O} / \mathrm{H}$ diagram. This results in $12+\log (\mathrm{O} / \mathrm{H})_{P}=7.74$ for object \#30 (as compared to $12+\log (\mathrm{O} / \mathrm{H})=7.86$ found by Contini et al. (2002) using the calibration of McGaugh 1991) and $\log (\mathrm{N} / \mathrm{O})_{P}=-1.58$ (as compared to $\log (\mathrm{N} / \mathrm{O})=-1.78$ found by Contini et al. 2002). 
The application of the $P$-method to the \#46 object results in $12+\log (\mathrm{O} / \mathrm{H})_{P}=8.07(12+\log (\mathrm{O} / \mathrm{H})=7.99$ was found by Contini et al. 2002). Thus this object does not belong to the lower branch of the $R_{23}-\mathrm{O} / \mathrm{H}$ diagram, and its abundances cannot be found through the $P$-method. It should be noted that the diagnostic line [OIII] $\lambda 4363$ should be detectable in lowmetallicity objects like \#30 and \#46 in better quality spectra.

Thus, the level of $\log (\mathrm{N} / \mathrm{O})=-1.6$ corresponds to the cumulative nitrogen to oxygen yields ratio in massive and intermediate-mass stars. At present time there are no firm arguments against the conclusion of Izotov \& Thuan (1999) that the nitrogen in low-metallicity blue compact galaxies has been produced by massive stars only and the level of $\log (\mathrm{N} / \mathrm{O})=-1.6$ is, in fact, defined by the nitrogen to oxygen yield ratio in massive stars. On the other hand, the constancy of N/O ratios in low-metallicity blue compact galaxies can be equally well reproduced under the assumption that a significant part of nitrogen is produced by intermediate-mass stars, i.e. the level of $\log (\mathrm{N} / \mathrm{O})=-1.6$ is defined by the cumulative nitrogen to oxygen yield ratio in massive and intermediate-mass stars (Pilyugin 1999). Therefore the adopted contributions of massive and intermediate-mass stars to the nitrogen production are based more on belief than on proof.

The principal result obtained here is that there is a longtime-delayed contribution to the nitrogen production by lowmass stars. It is this contribution that produces the high $\log (\mathrm{N} / \mathrm{O})>-1.6$ in some low-metallicity galaxies. Since the N/O ratio in the H II regions of the Small Magellanic Cloud is close to its lowest level, one can conclude that the bulk of the low-mass stars of the Small Magellanic Cloud, which are responsible for the long-time-delayed contribution to the nitrogen production, have not had enough time to evolve. Pagel \& Tautvaišiene (1998) have found that the age-metallicity relation for the Small Magellanic Cloud is well reproduced by a model in which the majority of the stars are under 4 Gyr old. In this case the low-mass stars with lifetimes longer than 4 Gyr are responsible for the long-time-delayed contribution to the nitrogen production. Recently Rich et al. (2000) have studied the age distribution of star clusters in the Small Magellanic Cloud. They have found that the clusters appear to have formed in two brief intervals, the oldest $8 \pm 2 \mathrm{Gyr}$ ago and a more recent burst $2 \pm 0.5 \mathrm{Gyr}$ ago. If the history of cluster formation reflects the total star formation history in the Small Magellanic Cloud, then the low-mass stars with lifetimes longer than $8 \pm 2$ Gyr are responsible for the long-time-delayed contribution to the nitrogen production.

As noted above, it is now commonly accepted now that the increase of the $\mathrm{N} / \mathrm{O}$ with $\mathrm{O} / \mathrm{H}$ at higher metallicities is caused by the metallicity-dependent nitrogen production in intermediate-mass stars in high-metallicity environment. However, one comment should be made. Henry et al. (2000) have fit their model to the $\mathrm{N} / \mathrm{O}$ versus $\mathrm{O} / \mathrm{H}$ trend for a sample of galaxies, and have found that nitrogen demonstrates a steeper dependence on metallicity than expected from a simple secondary behaviour. Thurston et al. (1996) have determined the slopes of a linear fit to the $\mathrm{N} / \mathrm{O}$ versus $\mathrm{O} / \mathrm{H}$ data for individual galaxies in their sample, and have found that $\mathrm{N} / \mathrm{O}$ is varying less steeply with metallicity, with slopes between 0.15 and 1.14, as compared with the 1.0 expected from a simple secondary behaviour. The slopes of a linear fit to the N/O versus $\mathrm{O} / \mathrm{H}$ data for individual galaxies in our sample show also a large spread (Fig. 5), but about 2/3 of the galaxies show slopes less 1.0. The shape of the N/O trend predicted by the model of Henry et al. (2000) corresponds well to the shape of the lower envelope exhibited by the data (see their Fig. 2b). However, close examination of our Fig. 5 clearly shows that the N/O vs. $\mathrm{O} / \mathrm{H}$ trends for individual galaxies do not correspond well to the shape of the lower envelope for all galaxies. Thus, the use of a mix of data including galaxies with different star formation histories - and consequently, with different histories of heavy element enrichment - can result in misleading conclusions on the behaviour of $\mathrm{N} / \mathrm{O}$ with $\mathrm{O} / \mathrm{H}$.

The value of $\mathrm{N} / \mathrm{O}$ for a given $\mathrm{O} / \mathrm{H}$ and the behaviour of $\mathrm{N} / \mathrm{O}$ with $\mathrm{O} / \mathrm{H}$ across the galaxy contain important information about the history of enrichment of that galaxy in heavy elements (and consequently, about the star formation history). This information is necessary for the construction of reliable models for the chemical evolution of galaxies. In turn, the construction of detailed models for galaxies of different morphological types can help to define more precisely the sites of nitrogen production. The construction of models for the chemical evolution of galaxies of different morphological types will be carried out in future studies.

\section{Conclusions}

The problem of the origin of nitrogen has been considered within the framework of an empirical approach. The oxygen abundances and nitrogen to oxygen abundances ratios have been derived in $\mathrm{H}_{\text {II }}$ regions of a number of spiral galaxies using the recently suggested $P$-method for more than six hundred published spectra. The $\mathrm{N} / \mathrm{O}-\mathrm{O} / \mathrm{H}$ diagram for $\mathrm{H}$ II regions in irregular and spiral galaxies has then been constructed.

It has been found that the level of $\log (\mathrm{N} / \mathrm{O})=-1.6$ corresponds to the cumulative nitrogen and oxygen productions by massive and intermediate-mass stars. The constancy of N/O ratios in low-metallicity galaxies can be as well reproduced under the assumption that massive stars make a dominant contribution to the nitrogen production in low-metallicity environments, as under the assumption that a significant part of nitrogen is produced by intermediate-mass stars. The available data do not allow to decide between these two possibilities.

It has been found that the $\mathrm{N} / \mathrm{O}$ values in $\mathrm{H}$ in regions of spiral galaxies of early morphological types are higher than those in H II regions with the same metallicity in spiral galaxies of late morphological types. This suggests that there is a long-time-delayed contribution to the nitrogen production. The scatter in $\mathrm{N} / \mathrm{O}$ values at a given $\mathrm{O} / \mathrm{H}$ can be naturally explained by differences in star formation histories in galaxies. The low-metallicity dwarf galaxies with $\mathrm{N} / \mathrm{O}$ close to the level of $\log (\mathrm{N} / \mathrm{O})=-1.6$ do not contain an appreciable amount of old stars. The long-time-delayed contribution to the nitrogen production by old low-mass stars is responsible for the high $\log (\mathrm{N} / \mathrm{O})>-1.6$ in some low-metallicity galaxies. 
The N/O ratio of a galaxy is an indicator of the time that has elapsed since the bulk of star formation occurred, or in other words of the nominal "age" of the galaxy as suggested by Edmunds \& Pagel (1978).

Consideration of the planetary nebulae in the Small Magellanic Cloud and the Milky Way Galaxy suggests that the contribution of low-mass stars to the nitrogen production is appreciable, and at low metallicities the contribution of lowmass stars can exceed the contribution of the intermediate-mass stars. This confirms our conclusion, reached from the consideration of the $\mathrm{N} / \mathrm{O}-\mathrm{O} / \mathrm{H}$ diagram for spiral galaxies of different morphological types, that there is a long-time-delayed contribution to the nitrogen production.

Acknowledgements. We thank Y. Izotov and J. Köppen for useful discussions. We thank the referee, G.Meynet, for constructive comments. This study was partly supported (L.S.P.) by the Joint Research Project between Eastern Europe and Switzerland (SCOPE) No. 7UKPJ62178, the NATO grant PST.CLG.976036, and the Italian national grant delivered by the MURST.

\section{References}

Aller, L. H., \& Czyzak, S. 1983, ApJS, 51, 211

Aller, L. H., \& Keyes, C. D. 1987, ApJS, 65, 405

Alloin, D., Edmunds, M. G., Lindblad, P. O., \& Pagel, B. E. J. 1981, A\&A, 101, 377

Blair, W. P., Kirshner, R. P., \& Chevalier, R. A. 1982, ApJ, 254, 50

Boothroyd, A. I., \& Sackmann, I.-J. 1999, ApJ, 510, 232

Bresolin, F., Kennicutt, R. C., \& Garnett, D. R. 1999, ApJ, 510, 104

Cahn, J. H., Kaler, J. B., \& Stanghellini, L. 1992, A\&AS, 94, 399

Castellanos, M., Diáz, A. I., \& Terlevich, E. 2002, MNRAS, 329, 315

Contini, T., Treyer, M. A., Sullivan, M., \& Ellis, R. S. 2002, MNRAS, 330,75

Costa, R. D. D., de Freitas Pacheco, J. A., \& Idiart, T. P. 2000, A\&ASS, 145, 467

Coziol, R., Carlos Reyes, R. E., Considére, S., Davoust, E., \& Contini, T. 1999, A\&A, 345, 733

Cunha, K., \& Lambert, D. L. 1992, ApJ, 399, 586

Cunha, K., \& Lambert, D. L. 1994, ApJ, 426, 170

Dennefeld, M., \& Kunth, D. 1981, AJ, 86, 989

Diaz, A. I., Terlevich, E., Vilchez, J. M., Pagel, B. E. J., \& Edmunds, M. G. 1991, MNRAS, 253, 245

Dufour, R. J. 1975, ApJ, 195, 315

Dufour, R. J., \& Harlow, W. V. 1977, ApJ, 216, 706

Dufour, R. J., Talbot, R. J., Jensen, E. B., \& Shields, G. A. 1980, ApJ, 236, 119

Edmunds, M. G., \& Pagel, B. E. J. 1978, MNRAS, 185, 77P

Edmunds, M. G., \& Pagel, B. E. J. 1984, MNRAS, 211, 507

Ferguson, A. M. N., Gallagher, J. S., \& Wyse, R. F. G. 1998, AJ, 116, 673

Fierro, J., Torres-Peimbert, S., \& Peimbert, M. 1986, PASP, 98, 1032

Garnett, D. R. 1990, ApJ, 363, 142

Garnett, D. R. 1992, AJ, 103, 1330

Garnett, D. R., \& Kennicutt, R. C. 1994, ApJ, 426, 123

Garnett, D. R., \& Shields, G. A. 1987, ApJ, 317, 82

Garnett, D. R., Shields, G. A., Peimbert, M., et al. 1999, ApJ, 513, 168

Garnett, D. R., Shields, G. A., Skillman, E. D., Sagan, S. P., \& Dufour, R. J. 1997, ApJ, 489, 63

Henry, R. B. C., Balkowski, C., Cayatte, V., Edmunds, M. G., \& Pagel, B. E. J. 1996, MNRAS, 283, 635

Henry, R. B. C., Edmunds, M. G., \& Köppen, J. 2000, ApJ, 541, 660
Henry, R. B. C., Pagel, B. E. J., \& Chincarini, G. L. 1994, MNRAS, 266,421

Henry, R. B. C., Pagel, B. E. J., Lasseter, D. F., \& Chincarini, G. L. 1992, MNRAS, 258, 321

Hidalgo-Gámez, A. M., \& Olofsson, K. 1998, A\&A, 334, 45

Izotov, Y. I., Chaffe, F. H., \& Green, R. F. 2001, ApJ, 562, 727

Izotov, Y. I., \& Thuan, T. X. 1999, ApJ, 511, 639

Kennicutt, R. C., \& Garnett, D. R. 1996, ApJ, 456, 504

Kingsburgh, R. L., \& Barlow, M. J. 1994, MNRAS, 271, 257

Kingsburgh, R. L., \& English, J. 1992, MNRAS, 259, 635

Kinkel, U., \& Rosa, M. R. 1994, A\&A, 282, L37

Kobulnicky, H. A., \& Skillman, E. D. 1996, ApJ, 471, 211

Kobulnicky, H. A., \& Skillman, E. D. 1997, ApJ, 489, 636

Kobulnicky, H. A., \& Skillman, E. D. 1998, ApJ, 497, 601

Köppen, J., Acker, A., \& Stenholm, B. 1991, A\&A, 248, 197

Kunth, D., \& Sargent, W. L. W. 1986, ApJ, 300, 496

Kwitter, K. B., \& Aller, L. H. 1981, MNRAS, 195, 939

Leisy, P., \& Dennefeld, M. 1996, A\&ASS, 116, 95

Leisy, P., \& Dennefeld, M. 2000, Rev. Mex Astron. Astrofis. (Conf. Ser.), 9, 227

Lequeux, J., Peimbert, M., Rayo, J. F., Serrano, A., \& Torres-Peimbert, S. 1979, A\&A, 80, 155

Lu, L., Sargent, W. L. W., \& Barlow, T. A. 1998, ApJ, 115, 55

Maciel, W. J. 1984, A\&AS, 55, 253

Maeder, A. 1992, A\&A, 264, 105

Marconi, G., Matteucci, F., \& Tosi, M. 1994, MNRAS, 270, 35

Marigo, P. 2001, A\&A, 370, 194

Marigo, P., Bressan, A., \& Chiosi, C. 1996, A\&A, 313, 545

Marigo, P., Bressan, A., \& Chiosi, C. 1998, A\&A, 331, 564

Matteucci, F. 1986, MNRAS, 221, 911

Matteucci, F., \& Tosi, M. 1985, MNRAS, 217, 391

McCall, M. L., Rybski, P. M., \& Shields, G. A. 1985, ApJS, 57, 1

McGaugh, S. S. 1991, ApJ, 380, 140

Meynet, G., \& Maeder, A. 2002a, A\&A, 381, L25

Meynet, G., \& Maeder, A. 2002b, A\&A, 390, 561

Mighell, K. J., Sarajedini, A., \& French, R. C. 1998, AJ, 116, 2395

Milingo, J. B., Henry, R. B. C., \& Kwitter, K. B. 2002, ApJS, 138, 285

Pagel, B. E. J., Edmunds, M. G., Blackwell, D. E., Chun, M. S., \& Smith, G. 1979, MNRAS, 189, 95

Pagel, B. E. J., Edmunds, M. G., Fosbury, R. A. E., \& Webster, B. L. 1978, MNRAS, 184, 569

Pagel, B. E. J., Simonson, E. A., Terlevich, R. J., \& Edmunds, M. G. 1992, MNRAS, 255, 325

Pagel, B. E. J., \& Tautvaišiene, G. 1998, MNRAS, 299, 535

Peimbert, M. 1978, in IAU Symp. 76, Planetary Nebulae: Observations and Theory, ed. Y. Terzian (Dordrecht: Reidel), 215

Peimbert, M., Peimbert, A., \& Ruiz, M. T. 2000, ApJ, 541, 688

Peimbert, M., \& Torres-Peimbert, S. 1976, ApJ, 203, 581

Pettini, M., Lipman, K., \& Hunstead, R. W. 1995, ApJ, 451, 100

Pettini, M., Ellison, S. L., Bergeron, J., \& Petitjean, P. 2002, A\&A, 391, 21

Pilyugin, L. S. 1992, A\&A, 260, 58

Pilyugin, L. S. 1993, A\&A, 277, 42

Pilyugin, L. S. 1999, A\&A, 346, 428

Pilyugin, L. S. 2000, A\&A, 362, 325

Pilyugin, L. S. 2001a, A\&A, 369, 594

Pilyugin, L. S. 2001b, A\&A, 373, 56

Pilyugin, L. S. 2001c, A\&A, 374, 412

Pilyugin, L .S., \& Edmunds, M. G. 1996a, A\&A, 313, 783

Pilyugin, L. S., \& Edmunds, M. G. 1996b, A\&A, 313, 792

Pilyugin, L. S., Ferrini, F., \& Shkvarun, R. V. 2002, in preparation

Prochaska, J. X., Henry, R. B. C., O’Meara, J. M., et al. 2002, PASP, 114,933 
Rauscher, T., Heger, A., Hoffman, R. D., \& Woosley, S. E. 2002, ApJ, 576, 323

Rayo, J. F., Peimbert, M., \& Torres-Peimbert, S. 1982, ApJ, 255, 1

Renzini, A., \& Voli, M. 1981, A\&A, 94, 175

Rich, R. M., Shara, M., Fall, S. M., \& Zubek, D. 2000, ApJ, 119, 197

Richer, M. G. 1993, ApJ, 415, 240

Roy, J.-R., \& Walsh, J. R. 1997, MNRAS, 288, 715

Sandage, A. 1986, A\&A, 161, 89

Searle, L. 1971, ApJ, 168, 327

Serrano, A., \& Peimbert, M. 1983, Rev. Mex. Astrno. Astrofis, 8, 117

Shields, G. A., \& Searle, L. 1978, ApJ, 222, 821

Shields, G. A., Skillman, E. D., \& Kennicutt, R. C. 1991, ApJ, 371, 82

Skillman, E. D., Kennicutt, R. C., Shields, G. A., \& Zaritsky, D. 1996, ApJ, 462, 147

Smith, H. E. 1975, ApJ, 199, 591

Staufer, J. R., \& Bothum, G. D. 1984, AJ, 89, 1702

Tenorio-Tagle, G. 1996, AJ, 111, 1641
Thuan, T. X., Izotov, Yu. I., \& Lipovetsky, V. A. 1995, ApJ, 445, 108 Thurston, T. R., Edmunds, M. G., \& Henry, R. B. C. 1996, MNRAS, 283, 990

Torres-Peimbert, S., \& Peimbert, M. 1997, in Planetary Nebulae, ed. H. J. Habing, \& H. J. G. L. M. Lamers (Dordrecht: Kluwer), IAU Symp., 180, 175

Torres-Peimbert, S., Peimbert, M., \& Fierro, J. 1989, ApJ, 345, 186

van den Hoek, L. B., \& Groenewegen, M. A. T. 1997, A\&AS, 123, 305

van Zee, L., Haynes, M. P., \& Salzer, J. J. 1997, AJ, 114, 2479

van Zee, L., Salzer, J. J., \& Haynes, M. P. 1998a, ApJ, 497, L1

van Zee, L., Salzer, J. J., Haynes, M. P., O'Donoghue, A. A., \& Balonek, T. J. 1998b, AJ, 116, 2805

Vila-Costas, M. B., \& Edmunds, M. G. 1993, MNRAS, 265, 199

Vilchez, J. M., Pagel, B. E. J., Diaz, A. I., Terlevich, E., \& Edmunds, M. G. 1988, MNRAS, 235, 633

Webster, B. L., \& Smith, M. G. 1983, MNRAS, 204, 743 